\title{
A mimetic spectral element solver for the Grad-Shafranov equation
}

\author{
A. Palha ${ }^{a, *}$, B. Koren ${ }^{b}$, F. Felici ${ }^{a}$ \\ a Eindhoven University of Technology, Department of Mechanical Engineering, P.O. Box 513, 5600 MB Eindhoven, The Netherlands \\ ${ }^{\mathrm{b}}$ Eindhoven University of Technology, Department of Mathematics and Computer Science, P.O. Box 513, 5600 MB Eindhoven, The Netherlands
}

\section{A R T I C L E I N F O}

\section{Article history:}

Received 1 December 2015

Received in revised form 28 March 2016

Accepted 2 April 2016

Available online 8 April 2016

\section{Keywords:}

Grad-Shafranov

Spectrally accurate

Spectral element

Poisson solver

Mimetic discretization

Mixed finite element

\begin{abstract}
A B S T R A C T
In this work we present a robust and accurate arbitrary order solver for the fixed-boundary plasma equilibria in toroidally axisymmetric geometries. To achieve this we apply the mimetic spectral element formulation presented in [56] to the solution of the GradShafranov equation. This approach combines a finite volume discretization with the mixed finite element method. In this way the discrete differential operators $(\nabla, \nabla \times, \nabla \cdot)$ can be represented exactly and metric and all approximation errors are present in the constitutive relations. The result of this formulation is an arbitrary order method even on highly curved meshes. Additionally, the integral of the toroidal current $J_{\phi}$ is exactly equal to the boundary integral of the poloidal field over the plasma boundary. This property can play an important role in the coupling between equilibrium and transport solvers. The proposed solver is tested on a varied set of plasma cross sections (smooth and with an X-point) and also for a wide range of pressure and toroidal magnetic flux profiles. Equilibria accurate up to machine precision are obtained. Optimal algebraic convergence rates of order $p+1$ and geometric convergence rates are shown for Soloviev solutions (including high Shafranov shifts), field-reversed configuration (FRC) solutions and spheromak analytical solutions. The robustness of the method is demonstrated for non-linear test cases, in particular on an equilibrium solution with a pressure pedestal.
\end{abstract}

(c) 2016 Elsevier Inc. All rights reserved.

\section{Introduction}

The numerical computation of magnetohydrodynamic (MHD) equilibria plays a central role in the study of magnetically confined plasmas. In particular, MHD equilibria are used as input to complex algorithms capable of performing detailed simulations of MHD turbulence and stability, transport, heating, etc., see for example $[11,18,19,28,31,45,66]$. Other applications of MHD equilibrium computations that have been gaining an increasing attention are discharge scenario validation and control of tokamak reactors, see [36] for an overview of current and future applications in control. In the context of control and discharge scenario validation, MHD equilibria are typically used in coupled simulations with 1D transport codes, e.g. $[3,16,19,33,34,57]$. With these applications in mind, the development of fast, robust and accurate MHD equilibrium solvers on arbitrary geometries has become an important and active topic of research.

For plasmas in axisymmetric configuration, such as in tokamak devices, the MHD equilibrium can be expressed in cylindrical coordinates $(r, z, \phi)$ by the Grad-Shafranov equation, see [30,65]:

\footnotetext{
* Corresponding author.

E-mail address: a.palha@tue.nl (A. Palha).
} 


$$
-\frac{1}{\mu_{0} r} \frac{\partial^{2} \psi}{\partial r^{2}}+\frac{1}{\mu_{0} r^{2}} \frac{\partial \psi}{\partial r}-\frac{1}{\mu_{0} r} \frac{\partial^{2} \psi}{\partial z^{2}}=r \frac{\mathrm{d} P}{\mathrm{~d} \psi}+\frac{1}{\mu_{0} r} f \frac{\mathrm{d} f}{\mathrm{~d} \psi} \quad \text { in } \quad \Omega_{p},
$$

where $\psi$ is the flux function, $f$ is related to the toroidal component of the magnetic flux, $P$ is the plasma pressure and where $\Omega_{p}=\Omega_{p}(\psi)$ denotes the plasma domain. For a complete derivation of this equation see for example the book by Goedbloed et al. [26].

The Grad-Shafranov equation, (1), is a non-linear elliptic partial differential equation. Its non-linear character stems from the non-linear dependence of $P$ and $f$ on the unknown flux function $\psi$ and from the fact that the plasma domain $\Omega_{p}$ is also an unknown, that in general can only be determined once the flux function is known. These two characteristics make the solution of this equation a challenging task.

The literature on the numerical solution of MHD equilibria is extensive. A detailed review of different formulations and codes prior to 1991 is presented in [67] and a shorter review up to 1984 is given in [5]. More recently, several other approaches have been proposed and are used by different research groups, e.g. CEDRES++ [32], CHEASE [50,51], CREATE-NL+ [2], ECOM [46,59], EEC [47], ESC [71], HELENA [37], NIMEQ [35], SPIDER [40], etc.

The solution of MHD equilibria can be grouped into two distinct classes: (i) fixed-boundary (e.g. CHEASE, ECOM, EEC, ESC, HELENA, NIMEQ SPIDER) and (ii) free-boundary (e.g. CEDRES++, CREATE-NL+). In the fixed-boundary case the plasma domain is prescribed together with $\psi=$ constant at its boundary, $\partial \Omega_{p}$. Equation (1) is then used to find $\psi$ inside the plasma. The free-boundary approach requires the solution of the MHD equilibrium in an infinite domain with homogeneous boundary conditions, $\psi=0$, at infinity and taking into consideration the current flowing in a set of external coils and the Grad-Shafranov equation in the plasma region, (1). In this situation, both the plasma domain and the flux function, $\psi$, are unknowns that need to be computed consistently. Both in the fixed- and free-boundary cases, the functions $P(\psi)$ and $f(\psi)$ are either prescribed or determined from transport codes (e.g. ASTRA [60], CORSICA [17], CRONOS [3], JETTO [14], RAPTOR [20], TRANSP [13]).

The different schemes to numerically solve the MHD equilibrium problem can either compute the flux function, $\psi$, on a prescribed mesh in the $(r, z)$ coordinate system (Eulerian or direct solvers, e.g. CEDRES++, CHEASE, CREATE-NL+, ECOM, $[29,39,41])$ or employ a flux-based mesh and compute the physical coordinates $(r, z)$ from the plasma geometry and $\psi$ (Lagrangian or indirect solvers, e.g. EEC, ESC, $[42,49])$. Regarding the numerical formulation, several different approaches have been proposed: finite element method (CEDRES++, CHEASE, CREATE-NL+, EEC, ESC, HELENA, NIMEQ [41]), spectral element or collocation method (ECOM, ESC, NIMEQ [49]), finite difference method [29] and radial-basis function meshfree method [39].

In this article we present a new arbitrary order, fixed-boundary, Eulerian MHD equilibrium solver based on a mimetic spectral element method formulation, [56]. High-order accuracy is obtained by (i) reformulating the Grad-Shafranov equation, (1), as a non-linear scalar Poisson equation with a non-uniform tensorial material property in the constitutive equation (see for example [53] for a derivation of Darcy's law), (ii) using a mixed finite element formulation for the equations containing metric and material properties and a finite volume formulation for the equations establishing topological relations, and (iii) using a particular set of arbitrary order finite element basis functions (edge basis functions, see [22,56]). To our knowledge, the proposed approach results in the first spectrally accurate Grad-Shafranov solver capable of reconstructing the total plasma current on highly curved meshes. An important characteristic of this solver is that it can account for arbitrary plasma shapes, including plasma shapes with an X-point. Also, no restrictions on the flux functions $f(\psi)$ and $P(\psi)$ are required, allowing for the computation of a wide variety of equilibria.

Mimetic methods aim to preserve essential physical/mathematical structures in a discrete setting. Many of such structures are topological, i.e. independent of metric, and involve integral relations. For these reasons the mimetic method uses an integral formulation in order to preserve these properties at the discrete level. A general presentation of the mimetic formulation used in this work is given in [56] and earlier work is presented in [10,23,24,54,55]. For other mimetic formulations see the references in [56].

The outline of this paper is as follows. In Section 2 we present the proposed numerical method. We start by introducing the Grad-Shafranov equation as a non-linear Poisson equation in Section 2.1 and then in Section 2.2 the iterative procedure used is discussed. This is followed by the mimetic discretization of the linear Poisson equation in Section 2.3. We finalize the presentation of the method by applying the Poisson solver to the discretization of the Grad-Shafranov equation. In Section 3 the proposed method is applied and tested on a varied set of test cases. We start by testing algebraic and geometric convergence rates on Soloviev (Section 3.1.1), field-reversed (Section 3.1.2) and spheromak (Section 3.1.3) analytical solutions. We then show the robustness of the method for linear and non-linear eigenvalue test cases, Section 3.2.2 and Section 3.2.3 respectively. In Section 4 we conclude with a discussion of the merits and limitations of this solver and future extensions.

\section{Numerical method}

\subsection{The Grad-Shafranov equation as a Poisson equation}

As stated before, the work presented here is focused on the fixed-boundary solution of the Grad-Shafranov equation, which corresponds to a homogeneous Dirichlet problem given by: 


$$
\begin{cases}-\frac{1}{\mu_{0} r} \frac{\partial^{2} \psi}{\partial r^{2}}+\frac{1}{\mu_{0} r^{2}} \frac{\partial \psi}{\partial r}-\frac{1}{\mu_{0} r} \frac{\partial^{2} \psi}{\partial z^{2}}=r \frac{\mathrm{d} P}{\mathrm{~d} \psi}+\frac{1}{\mu_{0} r} f \frac{\mathrm{d} f}{\mathrm{~d} \psi} & \text { in } \Omega_{p}, \\ \psi=0 & \text { on } \partial \Omega_{p}\end{cases}
$$

Since we consider a fixed-boundary solution, $\Omega_{p}$ is known. In the same manner, both $P$ and $f$ are also given.

Another form of this equation, that is typically presented in the literature, is

$$
\begin{cases}-\nabla \cdot\left(\frac{1}{\mu_{0} r} \nabla \psi\right)=r \frac{\mathrm{d} P}{\mathrm{~d} \psi}+\frac{1}{\mu_{0} r} f \frac{\mathrm{d} f}{\mathrm{~d} \psi} & \text { in } \Omega_{p} \\ \psi=0 & \text { on } \partial \Omega_{p} .\end{cases}
$$

Although this expression is correct, we prefer to use an equivalent formulation that highlights the physical nature of the problem:

$$
\left\{\begin{array} { l l } 
{ \nabla \times ( \mathbb { K } \nabla \times \psi ) = J _ { \phi } } & { \text { in } \quad \Omega _ { p } , } \\
{ \psi = 0 } & { \text { on } \partial \Omega _ { p } , }
\end{array} \quad \text { where } \left\{\begin{array}{l}
\mathbb{K}:=\left[\begin{array}{cc}
\frac{1}{\mu_{0} r} & 0 \\
0 & \frac{1}{\mu_{0} r}
\end{array}\right], \\
J_{\phi}:=r \frac{\mathrm{d} P}{\mathrm{~d} \psi}+\frac{1}{\mu_{0} r} f \frac{\mathrm{d} f}{\mathrm{~d} \psi},
\end{array}\right.\right.
$$

and with $\nabla \times \psi:=\frac{\partial \psi}{\partial z} \vec{e}_{r}-\frac{\partial \psi}{\partial r} \vec{e}_{z}$. This form shows that this boundary-value problem can be seen as a non-linear vector Poisson problem in $2 \mathrm{D}$ with a non-uniform tensorial material property $\mathbb{K}$ in the constitutive relation. Additionally, the following two relations are explicitly expressed in (4):

$$
\mathbb{K} \nabla \times \psi=\vec{h}_{\mathrm{p}}=h_{r} \vec{e}_{r}+h_{z} \vec{e}_{z} \quad \text { and } \quad \nabla \times \vec{h}_{\mathrm{p}}=J_{\phi}
$$

with $\vec{h}_{\mathrm{p}}$ the poloidal component of the magnetic field. By posing the fixed-boundary Grad-Shafranov problem as a non-linear Poisson problem we can focus on the numerical solution of the more general Poisson problem and then substitute $\mathbb{K}$ and $J_{\phi}$ by the particular cases present in MHD equilibria.

\subsection{Iterative solution of non-linear Poisson problem}

The solution of the non-linear Poisson problem (4) requires an iterative procedure such as Newton's method or a more straightforward fixed-point iteration method. In this work we focus on the fixed-point iteration scheme. It is important to note that the fixed-point iteration procedure does not converge in all cases. In the future a Newton method will be required as a more robust solver. Under some conditions (4) becomes an eigenvalue problem. In this situation a modification to the standard fixed-point iteration method is required. For this reason, we present first the non-eigenvalue case and then the eigenvalue one.

\subsubsection{Non-eigenvalue case}

With this simple method the updated value of the flux function, $\psi^{k+1}$, is computed by solving the Poisson problem with the right-hand side evaluated at the previous value $\psi^{k}$, that is $J_{\phi}\left(\psi^{k}, r, z\right)$. This means that for each iteration $k$ a linear Poisson problem is solved:

$$
\begin{cases}\nabla \times\left(\mathbb{K} \nabla \times \psi^{k+1}\right)=J_{\phi}\left(\psi^{k}, r, z\right) & \text { in } \Omega_{p}, \\ \psi=0 & \text { on } \partial \Omega_{p}\end{cases}
$$

The iterative procedure is stopped once the residual error satisfies

$$
\left\|\nabla \times\left(\mathbb{K} \nabla \times \psi^{k+1}\right)-J_{\phi}\left(\psi^{k+1}, r, z\right)\right\|_{L^{s}}<\epsilon \ll 1,
$$

with $\|\cdot\|_{L^{s}}$ the standard $s$-norm given by

$$
\|f\|_{L^{s}}:=\left(\int_{\Omega_{p}}|f|^{s} \mathrm{~d} V\right)^{\frac{1}{s}}
$$

and $s \in \mathbb{N}$. 


\subsubsection{Eigenvalue case}

In some situations the current profile $J_{\phi}$ has the form

$$
J_{\phi}(\psi, r, z)=\tilde{J}_{\phi}(\psi, r, z) \psi .
$$

In this case $\psi=0$ is a trivial solution of this equation and therefore the iterative procedure outlined above needs to be adapted in order to recover the physically relevant solution. Under this condition, the non-linear Poisson problem (4) becomes an eigenvalue problem, see for example $[25,43,48,59,64]$. Below we outline the procedure presented in [59], and followed in this work, for the solution of this eigenvalue problem.

The main idea is that it is possible to rescale the flux function, $\bar{\psi}:=\psi\|\psi\|_{L^{s}}^{-1}$, and the total toroidal current, $\bar{J}_{\phi}:=$ $J_{\phi} \sigma^{-1}$. Introducing these rescalings into $P, f$ and $J_{\phi}$ we get:

$$
\frac{\mathrm{d} P}{\mathrm{~d} \psi}=\frac{1}{\|\psi\|_{L^{s}}} \frac{\mathrm{d} P}{\mathrm{~d} \bar{\psi}}, \quad f \frac{\mathrm{d} f}{\mathrm{~d} \psi}=\frac{1}{2} \frac{\mathrm{d} f^{2}}{\mathrm{~d} \psi}=\frac{1}{\|\psi\|_{L^{s}}} \frac{\mathrm{d} f^{2}}{\mathrm{~d} \bar{\psi}} \quad \text { and } \quad J_{\phi}(\psi, r, z)=\frac{\sigma}{\|\psi\|_{L^{s}}} \bar{J}_{\phi}(\bar{\psi}, r, z) .
$$

Using the rescaling (8) results in the following non-linear eigenvalue problem:

$$
\begin{cases}\nabla \times(\mathbb{K} \nabla \times \bar{\psi})=\bar{\sigma} \bar{J}_{\phi} & \text { in } \quad \Omega_{p}, \\ \bar{\psi}=0 & \text { on } \partial \Omega_{p},\end{cases}
$$

with $\bar{\sigma}:=\frac{\sigma}{\|\psi\|_{L^{s}}^{2}}$ representing the eigenvalue. This form of the MHD equilibrium equation clearly shows that it is an eigenvalue problem on the eigenfunction $\bar{\psi}$ and associated eigenvalue $\bar{\sigma}$. This, as noted in [48,59], demonstrates the well-known scale-invariance property of the Grad-Shafranov equation under the transformation

$$
(\psi, \sigma, r, z) \longrightarrow\left(\lambda \psi, \lambda^{2} \sigma, r, z\right) .
$$

The fixed-point iterative procedure to find the physically relevant eigenfunction of this non-linear eigenvalue problem can either be employed to find the normalized flux function solution or the solution that satisfies a specific total toroidal current. Here we use the approach for finding a normalized flux function solution as presented in [59]. This method computes in each iteration a new eigen-pair $\left(\bar{\psi}^{k+1}, \bar{\sigma}^{k+1}\right)$ by first using the previous eigen-pair $\left(\bar{\psi}^{k}, \bar{\sigma}^{k}\right)$ to solve the linear Poisson problem:

$$
\begin{cases}\nabla \times\left(\mathbb{K} \nabla \times \psi^{k+1}\right)=\bar{\sigma}^{k} \bar{J}_{\phi}\left(\bar{\psi}^{k}, r, z\right) & \text { in } \Omega_{p}, \\ \psi^{k+1}=0 & \text { on } \partial \Omega_{p} .\end{cases}
$$

The new eigen-pair is computed in the following way:

$$
\bar{\psi}^{k+1}=\frac{\psi^{k+1}}{\left\|\psi^{k+1}\right\|_{L^{s}}} \quad \text { and } \quad \bar{\sigma}^{k+1}=\frac{\bar{\sigma}^{k}}{\left\|\psi^{k+1}\right\|_{L^{s}}} .
$$

Once showed that the iterative procedure to solve the non-linear Poisson problem (4) (and consequently the GradShafranov problem (2)) relies heavily on the successive solution of a linear Poisson problem, we proceed with the discussion on the application of the mimetic spectral element discretization to the solution of the linear Poisson problem.

\subsection{Poisson problem and its discrete solution}

In this section we present the application of the mimetic spectral element discretization to the solution of the linear Poisson problem, such as the one appearing in the iterative solution of the Grad-Shafranov problem, (11):

$$
\begin{cases}\nabla \times(\mathbb{K} \nabla \times \psi)=J & \text { in } \Omega_{p}, \\ \psi=\psi_{b} & \text { on } \partial \Omega_{p} .\end{cases}
$$

Note that (i) $J=J(r, z)$ since it is a linear Poisson problem and (ii) $\psi_{b} \neq 0$. The general case of inhomogeneous boundary conditions is outlined since it will be necessary in the solution of the Soloviev test case.

We start by rewriting (13) as a system of first order equations:

$$
\begin{cases}\mathbb{K} \nabla \times \psi=\vec{h}_{p} & \text { in } \quad \Omega_{p}, \\ \nabla \times \vec{h}_{p}=J & \text { in } \quad \Omega_{p}, \\ \psi=\psi_{b} & \text { on } \quad \partial \Omega_{p} .\end{cases}
$$


In this form, it is possible to separate the topological laws (exact) from the metric-dependent (approximate) ones. The second expression, $\nabla \times \vec{h}_{p}=J$, is a topological law (a circuital law in particular) that relates the flux integral of the current density $J$ through a surface $\mathcal{N}$ to the line integral of $\vec{h}_{p}$ over the boundary of $\mathcal{N}$ :

$$
\int_{\partial \mathcal{N}} \vec{h}_{p} \cdot \mathrm{d} \vec{l}=\int_{\mathcal{N}} J \mathrm{~d} V
$$

where we have used Stokes' theorem to establish the relation: $\int_{\partial \mathcal{N}} \vec{h}_{p} \cdot \mathrm{d} \vec{l}=\int_{\mathcal{N}} \nabla \times \vec{h}_{p} \mathrm{~d} V$. The integral form (15) highlights the topological, metric-free nature of this equation. On the other hand, the first equation, $\mathbb{K} \nabla \times \psi=\vec{h}_{p}$, is an approximate relation since it combines a topological relation, $\nabla \times \psi=\vec{b}_{p}$, with a constitutive one, $\mathbb{K} \vec{b}_{p}=\vec{h}_{p}$. Constitutive relations establish connections between different physical quantities by means of (inexact) physical constants and metric-dependent relations, as in this case where $\mathbb{K}$ is the metric-dependent tensorial material property. For these reasons, this equation has a local (metric-dependent) and approximate character opposed to the exact nature of the previously discussed topological law. For a detailed discussion of the nature of physical laws the authors advise the book by Tonti, [69].

It turns out, see for example [6-9,24,52,56,68-70] for an extensive discussion, that it is highly desirable (and in some situations essential) to exactly satisfy the topological equations at the discrete level, while all approximation and interpolation errors can be included in the constitutive relations.

With this objective in mind we will establish the mimetic discretization of the Poisson equation as expressed by the first order system (14). We start by recalling the standard inner product definitions for both scalar and vector valued functions,

$$
\langle g, f\rangle_{\Omega}:=\int_{\Omega} g f \mathrm{~d} V \quad \text { and } \quad\langle\vec{u}, \vec{v}\rangle_{\Omega}:=\int_{\Omega} \vec{u} \cdot \vec{v} \mathrm{~d} V,
$$

the associated norms,

$$
\|f\|_{L^{2}(\Omega)}:=\langle f, f\rangle_{\Omega}^{\frac{1}{2}} \quad \text { and } \quad\|\vec{u}\|_{L^{2}(\Omega)}:=\langle\vec{u}, \vec{u}\rangle_{\Omega}^{\frac{1}{2}},
$$

and the function spaces $L^{2}(\Omega)$ and $H(\nabla \times, \Omega)$,

$$
L^{2}(\Omega):=\left\{f \mid\|f\|_{L^{2}(\Omega)}<\infty\right\} \quad \text { and } \quad H(\nabla \times, \Omega):=\left\{\vec{u} \in L^{2}(\Omega) \mid \nabla \times \vec{u} \in L^{2}(\Omega)\right\} .
$$

Note that the $L^{2}(\Omega)$ space is defined for both scalar and vector valued functions.

The standard mixed finite element formulation starts by constructing the weak problem, see [12],

$$
\left\{\begin{array}{l}
\text { Find } \psi \in L^{2}\left(\Omega_{p}\right) \text { and } \vec{h}_{p} \in H\left(\nabla \times, \Omega_{p}\right) \text { such that } \\
\langle\psi, \nabla \times(\mathbb{K} \vec{\varphi})\rangle_{\Omega_{p}}=\left\langle\vec{h}_{p}, \vec{\varphi}\right\rangle_{\Omega_{p}}, \quad \forall \vec{\varphi} \in H\left(\nabla \times, \Omega_{p}\right) \\
\left\langle\nabla \times \vec{h}_{p}, \phi\right\rangle_{\Omega_{p}}=\langle J, \phi\rangle_{\Omega_{p}}, \quad \forall \phi \in L^{2}\left(\Omega_{p}\right) .
\end{array}\right.
$$

Note that we consider here homogeneous Dirichlet boundary conditions $\psi=\psi_{b}=0$ on $\partial \Omega_{p}$, which are now imposed weakly. Well-posedness of this weak formulation can be found in any book on mixed finite elements, e.g. [12].

This formulation has two shortcomings:

1. The discrete $\mathbb{K} \nabla \times$ operator will not be the adjoint of the discrete $\nabla \times$ operator, as opposed to the continuous case where both operators are adjoint. This can lead to the loss of self-adjointness and negative definiteness of the discrete Laplacian operator, $\nabla \times \mathbb{K} \nabla \times$, on general grids, leading to poor convergence properties, see for example [38].

2. The second equation, representing the topological relation, is satisfied only approximately in curved geometries, not exactly. We will see that with the use of a proper set of basis functions it is possible to write this equation in a purely topological form, which is exact even on curved geometries.

In order to overcome these two aspects of the standard mixed finite element formulation we will present the mimetic spectral element discretization. We start by introducing an alternative inner product, as discussed in [38,56,61], and show that this results in a $\mathbb{K} \nabla \times$ operator that is the adjoint of the $\nabla \times$ operator. Afterwards, we introduce the set of basis functions used to discretize the physical quantities and show that they result in an exact representation of the topological relation in (14).

\subsubsection{The natural inner product}

We can define an alternative inner product between two vector fields $\vec{u}, \vec{v} \in H(\nabla \times, \Omega)$ using the material metric tensor $\mathbb{K}$, since it is symmetric and positive definite. This inner product, which we denote by natural inner product, is defined by 


$$
\langle\vec{u}, \vec{v}\rangle_{\mathbb{K}, \Omega}:=\int_{\Omega}\left(\mathbb{K}^{-1} \vec{u}\right) \cdot \vec{v} \mathrm{~d} V=\int_{\Omega} \vec{u} \cdot\left(\mathbb{K}^{-1} \vec{v}\right) \mathrm{d} V
$$

If this inner product is used on the first equation in (18) we obtain the following alternative equality

$$
\langle\mathbb{K} \nabla \times \psi, \vec{\varphi}\rangle_{\mathbb{K}, \Omega_{p}}=\left\langle\vec{h}_{p}, \vec{\varphi}\right\rangle_{\mathbb{K}, \Omega_{p}}, \quad \forall \vec{\varphi} \in H\left(\nabla \times, \Omega_{p}\right) .
$$

Using the definition of the inner product, (19), and integrating by parts we obtain:

$$
\langle\mathbb{K} \nabla \times \psi, \vec{\varphi}\rangle_{\mathbb{K}, \Omega_{p}}:=\int_{\Omega_{p}}\left(\mathbb{K}^{-1} \mathbb{K} \nabla \times \psi\right) \cdot \vec{\varphi} \mathrm{d} V=\int_{\Omega_{p}} \psi \cdot \nabla \times \vec{\varphi} \mathrm{d} V-\int_{\partial \Omega_{p}} \psi \vec{\varphi} \cdot \mathrm{d} \vec{l}=\langle\psi, \nabla \times \vec{\varphi}\rangle-\int_{\partial \Omega_{p}} \psi \vec{\varphi} \cdot \mathrm{d} \vec{l}
$$

For homogeneous boundary conditions, $\psi_{b}=0$, this expression shows that the natural inner product between vector valued functions, (19), satisfies the adjoint relation between $\mathbb{K} \nabla \times$ and $\nabla \times$. In order to enforce this adjoint relation at the discrete level we rewrite the mixed finite element formulation, (18), as:

$$
\left\{\begin{array}{l}
\text { Find } \psi \in L^{2}\left(\Omega_{p}\right) \text { and } \vec{h}_{p} \in H\left(\nabla \times, \Omega_{p}\right) \text { such that } \\
\langle\psi, \nabla \times \vec{\varphi}\rangle_{\Omega_{p}}=\left\langle\vec{h}_{p}, \vec{\varphi}\right\rangle_{\mathbb{K}, \Omega_{p}}, \quad \forall \vec{\varphi} \in H\left(\nabla \times, \Omega_{p}\right), \\
\left\langle\nabla \times \vec{h}_{p}, \phi\right\rangle_{\Omega_{p}}=\langle J, \phi\rangle_{\Omega_{p}}, \quad \forall \phi \in L^{2}\left(\Omega_{p}\right) .
\end{array}\right.
$$

Note that the Dirichlet boundary conditions $\psi=\psi_{b}=0$ on $\partial \Omega_{p}$ are still imposed weakly. For inhomogeneous boundary conditions the boundary term $\int_{\partial \Omega_{p}} \psi \vec{\varphi} \cdot \mathrm{d} \vec{l}$ must be included.

\subsubsection{The finite-dimensional basis functions}

In this work we approximate $\psi$ and $\vec{h}_{p}$ by expanding them in two distinct families of tensor product polynomials of at most degree $p$ in $r$ and $z$ coordinates. First, two types of polynomials are introduced, one associated to nodal interpolation and the other associated to histopolation (see [22,62,63] for an extensive discussion of histopolation and its relation to integral interpolation). Subsequently, these two types of polynomials will be combined to generate the two-dimensional polynomial basis functions used to discretize $\psi$ and $\vec{h}_{p}$.

Consider the canonical interval $I=[-1,1] \subset \mathbb{R}$ and the Legendre polynomials, $L_{p}(\xi)$ of degree $p$ with $\xi \in I$. The $p+1$ roots, $\xi_{i}$, of the polynomial $\left(1-\xi^{2}\right) \frac{\mathrm{d} L_{p}}{\mathrm{~d} \xi}$ are called Gauss-Lobatto-Legendre (GLL) nodes and satisfy $-1=\xi_{0}<\xi_{1}<\cdots<$ $\xi_{p-1}<\xi_{p}=1$. Let $l_{i}^{p}(\xi)$ be the Lagrange polynomial of degree $p$ through the GLL nodes, such that

$$
l_{i}^{p}\left(\xi_{j}\right):=\left\{\begin{array}{ll}
1 & \text { if } i=j \\
0 & \text { if } i \neq j
\end{array}, \quad i, j=0, \ldots, p .\right.
$$

The explicit form of these Lagrange polynomials is given by

$$
l_{i}^{p}(\xi)=\prod_{\substack{k=0 \\ k \neq i}}^{p} \frac{\xi-\xi_{k}}{\xi_{i}-\xi_{k}}
$$

Let $q(\xi)$ be a function defined on $I$ and $q_{i}=q\left(\xi_{i}\right)$, then its expansion in terms of these polynomials, $q_{h}(\xi)$, is given by

$$
q_{h}(\xi):=\sum_{i=0}^{p} q_{i} l_{i}^{p}(\xi) .
$$

By $(23) q_{h}(\xi)$ is a polynomial interpolant of degree $p$ of $q(\xi)$. For this reason we denote the Lagrange polynomials in (24) by nodal polynomials.

Before introducing the second set of basis polynomials that will be used in this work it is important to introduce the reader to the concept of histopolant. Given a histogram, a histopolant is a function whose integrals over the cells (or bins) of the histogram are equal to the area of the corresponding bars of the histogram, see Fig. 1. If the histopolant is a polynomial we say that it is a polynomial histopolant. In the same way as a polynomial interpolant that passes exactly through $p+1$ points has degree $p$, a polynomial that exactly histopolates a histogram with $p+1$ bins has polynomial degree $p$. Consider now a function $g(x)$ and its associated integrals over a set of cells $\left[a_{j-1}, a_{j}\right], g_{j}=\int_{a_{j-1}}^{a_{j}} g(x) \mathrm{d} x$, with $a_{0},<\cdots<a_{j}<\cdots<a_{p}$. The set of integral values $g_{j}$ and cells $\left[a_{j-1}, a_{j}\right]$ can be seen as a histogram. As mentioned before, it is possible to construct a histopolant of this histogram. This histopolant will be an approximating function of $g$ that has the particular property of having the same integral over the cells $\left[a_{j-1}, a_{j}\right]$ as $g$. In the same way as an interpolant exactly reconstructs the original function at the interpolating points, a histopolant exactly reconstructs the integral of the original function over the cells. 


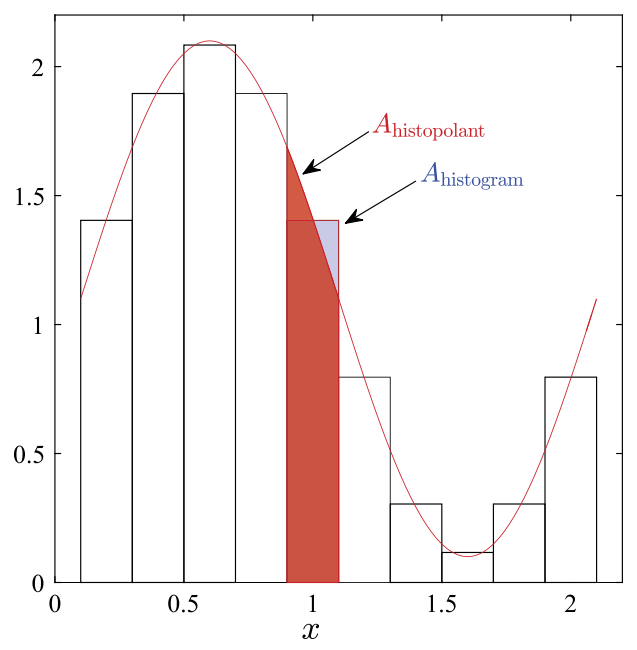

Fig. 1. Histogram and an example of a histopolant (red curve). By definition, the integral of the histopolant over each cell (or bin) $A_{\text {histopolant }}$ is equal to the area of the corresponding bar of the histogram $A_{\text {histogram. }}$ (For interpretation of the references to color in this figure legend, the reader is referred to the web version of this article.)

Using the nodal polynomials we can define another set of basis polynomials, $e_{i}^{p}(\xi)$, as

$$
e_{i}^{p}(\xi):=-\sum_{k=0}^{i-1} \frac{\mathrm{d} l_{k}^{p}(\xi)}{\mathrm{d} \xi}, \quad i=1, \ldots, p
$$

These polynomials $e_{i}^{p}(\xi)$ have polynomial degree $p-1$ and satisfy

$$
\int_{\xi_{j-1}}^{\xi_{j}} e_{i}^{p}(\xi) \mathrm{d} \xi=\left\{\begin{array}{ll}
1 & \text { if } i=j \\
0 & \text { if } i \neq j
\end{array}, \quad i, j=1, \ldots, p\right.
$$

The proof that the polynomials $e_{i}^{p}(\xi)$ have degree $p-1$ follows directly from the fact that their definition (26) involves a linear combination of the derivative of polynomials of degree $p$. The proof of (27) results from the properties of $l_{k}^{p}(\xi)$. Using (26) the integral of $e_{i}^{p}(\xi)$ becomes

$$
\int_{\xi_{j-1}}^{\xi_{j}} e_{i}^{p}(\xi) \mathrm{d} \xi=-\int_{\xi_{j-1}}^{\xi_{j}} \sum_{k=0}^{i-1} \frac{\mathrm{d} l_{k}^{p}(\xi)}{\mathrm{d} \xi}=-\sum_{k=0}^{i-1} \int_{\xi_{j-1}}^{\xi_{j}} \frac{\mathrm{d} l_{k}^{p}(\xi)}{\mathrm{d} \xi}=-\sum_{k=0}^{i-1}\left(l_{k}^{p}\left(\xi_{j}\right)-l_{k}^{p}\left(\xi_{j-1}\right)\right)=-\sum_{k=0}^{i-1}\left(\delta_{k, j}-\delta_{k, j-1}\right) .
$$

It is straightforward to see that

$$
-\sum_{k=0}^{i-1}\left(\delta_{k, j}-\delta_{k, j-1}\right)=\left\{\begin{array}{ll}
1 & \text { if } i=j \\
0 & \text { if } i \neq j
\end{array}, \quad i, j=1, \ldots, p .\right.
$$

For more details see $[22,62,63]$.

Let $g(\xi)$ be a function defined on $I$ and $g_{i}=\int_{\xi_{i-1}}^{\xi_{i}} g(\xi) \mathrm{d} \xi$, then its expansion in terms of these polynomials, $g_{h}(\xi)$, is given by

$$
g_{h}(\xi)=\sum_{i=1}^{p} g_{i} e_{i}^{p}(\xi)
$$

By (27) we have $\int_{\xi_{i-1}}^{\xi_{i}} g_{h}(\xi) \mathrm{d} \xi=g_{i}$ and therefore $g_{h}(\xi)$ is a polynomial histopolant of degree $p-1$ of $g(\xi)$. For this reason we denote the polynomials in (26) by histopolant polynomials.

It can be shown, [22,62], that if $q(\xi)$ is expanded in terms of nodal polynomials, as in (25), then the expansion of its derivative $\frac{\mathrm{d} q(\xi)}{\mathrm{d} \xi}$ in terms of histopolant polynomials is 

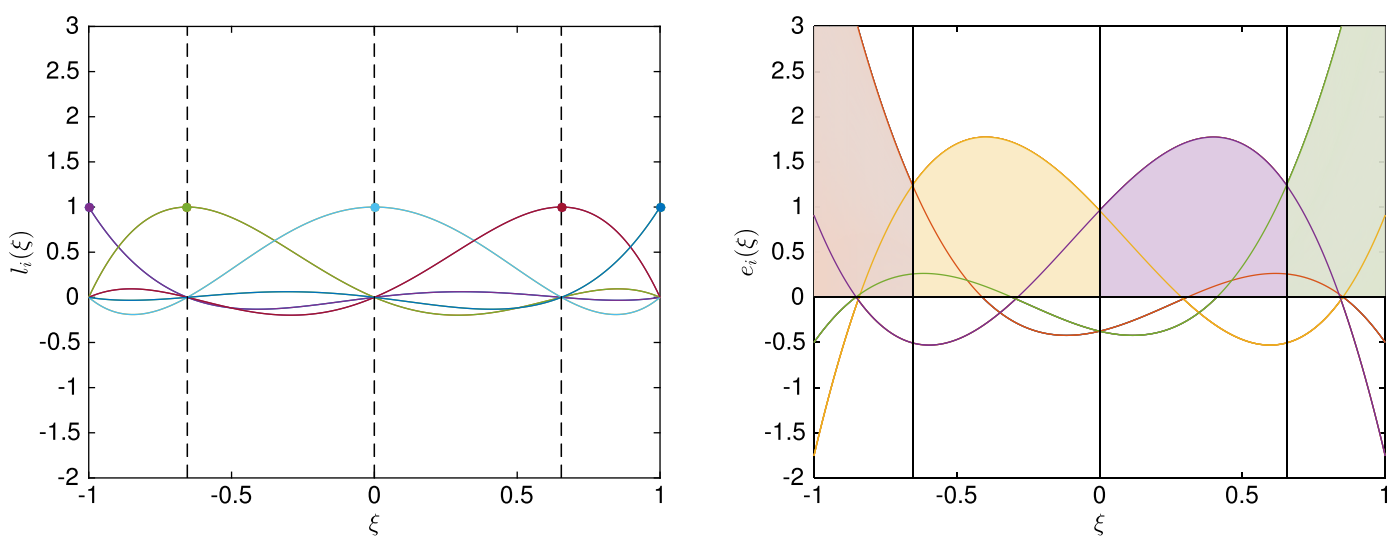

Fig. 2. Basis polynomials associated to $p=4$. Left: nodal polynomials, the value of the basis polynomial at the corresponding node is one and on the other nodes is zero. Right: histopolant polynomials, the integral of the basis polynomials over the corresponding shaded area evaluates to one and to zero on the others.

$$
\left(\frac{\mathrm{d} q(\xi)}{\mathrm{d} \xi}\right)_{h}=\sum_{i=1}^{p}\left(\int_{\xi_{i-1}}^{\xi_{i}} \frac{\mathrm{d} q(\xi)}{\mathrm{d} \xi} \mathrm{d} \xi\right) e_{i}^{p}(\xi)=\sum_{i=1}^{p}\left(q\left(\xi_{i}\right)-q\left(\xi_{i-1}\right)\right) e_{i}^{p}(\xi)=\sum_{i=1}^{p}\left(q_{i}-q_{i-1}\right) e_{i}^{p}(\xi)=\sum_{i=1, j=0}^{p} \mathrm{E}_{i, j}^{1,0} q_{j} e_{i}^{p}(\xi)
$$

where $E_{i, j}^{1,0}$ are the coefficients of the $p \times(p+1)$ matrix $\mathbf{E}^{1,0}$

$$
\mathbf{E}^{1,0}:=\left(\begin{array}{cccccc}
-1 & 1 & 0 & 0 & \ldots & 0 \\
0 & -1 & 1 & 0 & \ddots & 0 \\
\vdots & & \ddots & \ddots & & \vdots \\
0 & \ddots & 0 & -1 & 1 & 0 \\
0 & \ldots & 0 & 0 & -1 & 1
\end{array}\right),
$$

and the following identity holds

$$
\left(\frac{\mathrm{d} q(\xi)}{\mathrm{d} \xi}\right)_{h}=\frac{\mathrm{d} q_{h}(\xi)}{\mathrm{d} \xi}
$$

For an example of the basis polynomials corresponding to $p=4$ see Fig. 2 .

Combining histopolant polynomials we can construct the polynomial basis functions used to discretize $\psi$ on quadrilaterals. Consider the canonical interval $I=[-1,1]$, the canonical square $Q=I \times I \subset \mathbb{R}^{2}$, the histopolant polynomials (26), $e_{i}^{p}(\xi)$ of degree $p-1$, and take $\xi, \eta \in I$. Then a set of two-dimensional basis polynomials, $\omega_{k}^{p}(\xi, \eta)$, can be constructed as the tensor product of the one-dimensional ones

$$
\omega_{k}^{p}(\xi, \eta):=e_{i}^{p}(\xi) e_{j}^{p}(\eta), \quad i, j=1, \ldots, p, \quad k=j+(i-1) p .
$$

These polynomials, $\omega_{k}^{p}(\xi, \eta)$, have degree $p-1$ in each variable and satisfy, see [22,56],

$$
\int_{\xi_{i-1}}^{\xi_{i}} \int_{\eta_{j-1}}^{\eta_{j}} \omega_{k}^{p}(\xi, \eta) \mathrm{d} \xi \mathrm{d} \eta=\left\{\begin{array}{ll}
1 & \text { if } k=(i-1) p+j \\
0 & \text { if } k \neq(i-1) p+j
\end{array}, \quad i, j=1, \ldots, p, \quad k=1, \ldots, p^{2},\right.
$$

where, as before, $\xi_{i}$ and $\eta_{i}$ with $i=0, \ldots, p$ are the Gauss-Lobatto-Legendre (GLL) nodes. Let $\psi(\xi, \eta)$ be a function defined on $Q$ and $\psi_{k}=\int_{\xi_{i-1}}^{\xi_{i}} \int_{\eta_{j-1}}^{\eta_{j}} \psi(\xi, \eta) \mathrm{d} \xi \mathrm{d} \eta$ with $k=j+(i-1) p$, then its expansion in terms of these polynomials, $\psi_{h}(\xi, \eta)$, is given by

$$
\psi_{h}(\xi, \eta)=\sum_{k=1}^{p^{2}} \psi_{k} \omega_{k}^{p}(\xi, \eta)
$$


By (33) we have $\int_{\xi_{i-1}}^{\xi_{i}} \int_{\eta_{j-1}}^{\eta_{j}} \psi_{h}(\xi, \eta) \mathrm{d} \xi \mathrm{d} \eta=\psi_{k}$ for $k=j+(i-1) p$, with $i, j=1, \ldots, p$. Additionally, $\psi_{h}(\xi, \eta)$ is a twodimensional histopolant of $\psi(\xi, \eta)$ of degree $p-1$ in each variable. For this relation between the expansion coefficients and volume integration (in two dimensions volumes are surfaces) we denote the polynomials in (32) by volume polynomials. Moreover, these basis polynomials satisfy $\omega_{k}^{p}(\xi, \eta) \in L^{2}(Q)$.

In a similar way, but combining nodal polynomials with histopolant polynomials, we can construct the polynomial basis functions used to discretize $\vec{h}_{p}$ on quadrilaterals. Consider the nodal polynomials $(24), l_{i}^{p}(\xi)$ of degree $p$, the histopolant polynomials (26), $e_{i}^{p}(\xi)$ of degree $p-1$, and take $\xi, \eta \in I$. A set of two-dimensional basis polynomials, $\vec{\epsilon}_{k}^{p}(\xi, \eta)$, can be constructed as the tensor product of the one-dimensional ones

$$
\vec{\epsilon}_{k}^{p}(\xi, \eta):=\left\{\begin{array}{lll}
e_{i}^{p}(\xi) l_{j}^{p}(\eta) \vec{e}_{\xi} & \text { if } k<p(p+1), \quad \text { with } i=1, \ldots, p, \quad j=0, \ldots, p, \quad k=(i-1)(p+1)+j, \\
l_{i}^{p}(\xi) e_{j}^{p}(\eta) \vec{e}_{\eta} & \text { if } k \geq p(p+1), \quad \text { with } i=0, \ldots, p, \quad j=1, \ldots, p, \quad k=i p+j+p(p+1)-1 .
\end{array}\right.
$$

These polynomials, $\vec{\epsilon}_{k}^{p}(\xi, \eta)$, have degree $p-1$ in $\xi$ and $p$ in $\eta$ if $k<p(p+1)$. If $k \geq p(p+1)$ then the degree in $\xi$ is $p$ and the degree in $\eta$ is $p-1$. It is possible to show, see [22,56], that these polynomials satisfy

$$
\int_{\xi_{i-1}}^{\xi_{i}} \vec{\epsilon}_{k}^{p}\left(\xi, \eta_{j}\right) \cdot \vec{e}_{\xi} \mathrm{d} \xi=\left\{\begin{array} { l } 
{ 1 \quad \text { if } k = ( i - 1 ) ( p + 1 ) + j } \\
{ 0 \quad \text { if } k \neq ( i - 1 ) ( p + 1 ) + j }
\end{array} , \text { with } \left\{\begin{array}{l}
i=1, \ldots, p, \\
j=0, \ldots, p, \\
k=0, \ldots, 2 p(p+1)-1,
\end{array}\right.\right.
$$

and

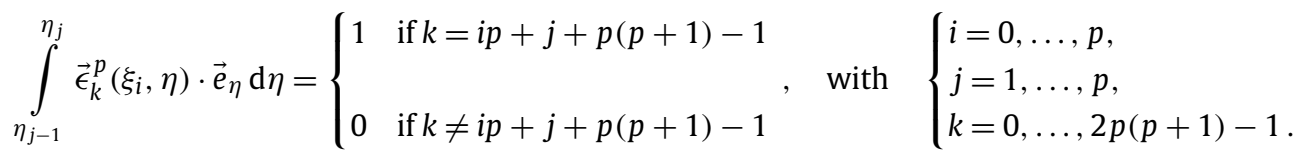

Let $\vec{h}_{p}(\xi, \eta)$ be a vector valued function defined on $Q$ and

$$
h_{p, k}=\left\{\begin{array}{l}
\int_{\xi_{i-1}}^{\xi_{i}} \vec{h}_{p}\left(\xi, \eta_{j}\right) \cdot \vec{e}_{\xi} \mathrm{d} \xi \quad \text { if } k<p(p+1), \quad \text { with } \quad\left\{\begin{array}{l}
i=1, \ldots, p, \\
j=0, \ldots, p, \\
k=(i-1)(p+1)+j,
\end{array}\right. \\
\int_{\eta_{j-1}}^{\eta_{j}} \vec{h}_{p}\left(\xi_{i}, \eta\right) \cdot \vec{e}_{\eta} \mathrm{d} \eta \quad \text { if } k \geq p(p+1), \quad \text { with } \quad\left\{\begin{array}{l}
i=0, \ldots, p, \\
j=1, \ldots, p, \\
k=i p+j+p(p+1)-1 .
\end{array}\right.
\end{array}\right.
$$

then its expansion in terms of these polynomials, $\vec{h}_{p, h}(\xi, \eta)$, is given by

$$
\vec{h}_{p, h}(\xi, \eta)=\sum_{k=0}^{2 p(p+1)-1} h_{p, k} \vec{\epsilon}_{k}^{p}(\xi, \eta),
$$

Using (36) we have

$$
\int_{\xi_{i-1}}^{\xi_{i}} \vec{h}_{p, h}\left(\xi, \eta_{j}\right) \cdot \vec{e}_{\xi} \mathrm{d} \xi=h_{p, k}, \text { for } k=(i-1)(p+1)+j, \text { with } i=1, \ldots, p \text { and } j=0, \ldots, p .
$$

In a similar way, using (37) we have

$$
\int_{\eta_{j-1}}^{\eta_{j}} \vec{h}_{p, h}\left(\xi_{i}, \eta\right) \cdot \vec{e}_{\eta} \mathrm{d} \eta=h_{p, k}, \text { for } k=i p+j+p(p+1)-1, \text { with } i=0, \ldots, p \text { and } j=1, \ldots, p .
$$

The expansion $\vec{h}_{p, h}(\xi, \eta)$ is a two-dimensional edge histopolant (interpolates line integrals) of $\vec{h}_{p}(\xi, \eta)$ with degrees $p-1$ in $\xi$ and $p$ in $\eta$ along the $\xi$ component and degrees $p$ in $\xi$ and $p-1$ in $\eta$ along the $\eta$ component. Since the coefficients of this expansion are edge (or line) integrals, we denote the polynomials in (35) by edge polynomials. Additionally, these basis polynomials satisfy $\vec{\epsilon}_{k}^{p}(\xi, \eta) \in H(\nabla \times, \Omega)$, see $[22,56]$. 

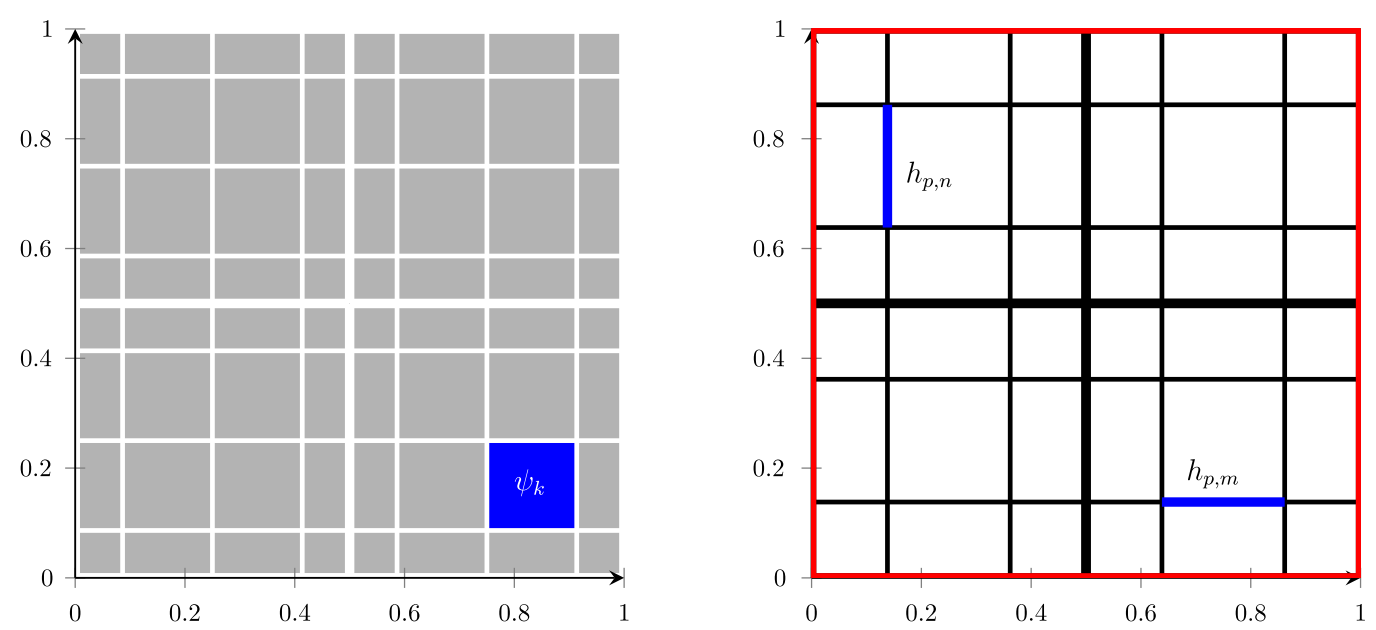

Fig. 3. Visual representation of the coefficients of the polynomial expansions using volume polynomials (left) and edge polynomials (right). These coefficients correspond to geometric degrees of freedom. For volume polynomials these geometric degrees of freedom are associated to surfaces of the spectral element mesh and for edge polynomials they are associated to edges of the spectral element mesh.

It can be shown, [22,56], that if $\vec{h}_{p}(\xi, \eta)$ is expanded in terms of edge polynomials, as in (39), then the expansion of $\nabla \times \vec{h}_{p}$ in terms of the volume polynomials, (32), is

$$
\begin{aligned}
&\left(\nabla \times \vec{h}_{p}(\xi, \eta)\right)_{h}=\sum_{i, j=1}^{p}\left(\int_{\xi_{i-1}}^{\xi_{i}} \int_{\eta_{j-1}}^{\eta_{j}} \nabla \times \vec{h}_{p}(\xi, \eta) \mathrm{d} \xi \mathrm{d} \eta\right) \omega_{j+(i-1) p}^{p}(\xi, \eta) \\
&=\sum_{i, j=1}^{p}\left(\int_{\xi_{i-1}}^{\xi_{i}} \vec{h}_{p}\left(\xi, \eta_{j-1}\right) \cdot \vec{e}_{\xi} \mathrm{d} \xi+\int_{\eta_{j-1}}^{\eta_{j}} \vec{h}_{p}\left(\xi_{i}, \eta\right) \cdot \vec{e}_{\eta} \mathrm{d} \eta\right. \\
& \stackrel{(38)}{=} \sum_{k=1}^{p^{2}}\left(h_{p, k+(k \operatorname{div} p)}+h_{p, k+p(p+2)}-h_{p, k+(k \operatorname{div} p)+1}-h_{p, k+p(p+1)}\right) \omega_{k}^{p}(\xi, \eta) \\
&\left.\left.=\int_{p^{2}, 2 p(p+1)-1}^{\xi_{i}} \vec{h}_{p}\left(\xi, \eta_{j}\right) \cdot \vec{e}_{\xi} \mathrm{d} \xi-\xi_{i-1}, \eta\right) \cdot \vec{e}_{\eta} \mathrm{d} \eta\right)_{j+(i-1) p}(\xi, \eta) \\
& \sum_{k=1, j=0}^{2,1} \mathrm{E}_{k, j} h_{p, j} \omega_{k}^{p}(\xi, \eta),
\end{aligned}
$$

where $\mathrm{E}_{k, j}^{2,1}$ are the coefficients of the $p^{2} \times 2 p(p+1)$ matrix $\mathbf{E}^{2,1}$ and are defined as

$$
\mathrm{E}_{k, j}^{2,1}:= \begin{cases}1 & \text { if } j=k+(k \operatorname{div} p), \\ 1 & \text { if } j=k+p(p+2), \\ -1 & \text { if } j=k+(k \operatorname{div} p)+1, \\ -1 & \text { if } j=k+p(p+1), \\ 0 & \text { otherwise. }\end{cases}
$$

The following identity holds

$$
\left(\nabla \times \vec{h}_{p}(\xi, \eta)\right)_{h}=\nabla \times \vec{h}_{p, h}
$$

Here, $(k \operatorname{div} p)$ denotes integer division, that is division in which the fractional part (remainder) is discarded, e.g. $(5$ div 3$)=1$, $(5 \operatorname{div} 2)=2$ and $(5 \operatorname{div} 6)=0$.

A graphical representation of the coefficients in the polynomial expansions, (34) and (39), is shown in Fig. 3. 


\subsubsection{Discrete representation of topological laws}

The finite-dimensional polynomial basis functions presented in Section 2.3.2 enable us to exactly satisfy the topological laws at a discrete level. Consider the topological law in (22)

$$
\left\langle\nabla \times \vec{h}_{p}, \phi\right\rangle_{\Omega_{p}}=\langle J, \phi\rangle_{\Omega_{p}}, \quad \forall \phi \in L^{2}\left(\Omega_{p}\right) .
$$

If $\vec{h}_{p}$ and $J$ are substituted by their polynomial expansions as in (39) and (34) respectively, using (42) the following expression is obtained

$$
\left\langle\sum_{k=1, j=0}^{p^{2}, 2 p(p+1)-1} \mathrm{E}_{k, j}^{2,1} h_{p, j} \omega_{k}^{p}(\xi, \eta), \phi\right\rangle_{\Omega_{p}}=\left\langle\sum_{k=1}^{p^{2}} J_{k} \omega_{k}^{p}(\xi, \eta), \phi\right\rangle_{\Omega_{p}}, \quad \forall \phi \in L^{2}\left(\Omega_{p}\right) .
$$

By linearity of the inner product this can be rewritten as

$$
\sum_{k=1, j=0}^{p^{2}, 2 p(p+1)-1} \mathrm{E}_{k, j}^{2,1} h_{p, j}\left\langle\omega_{k}^{p}(\xi, \eta), \phi\right\rangle_{\Omega_{p}}=\sum_{k=1}^{p^{2}} J_{k}\left\langle\omega_{k}^{p}(\xi, \eta), \phi\right\rangle_{\Omega_{p}}, \quad \forall \phi \in L^{2}\left(\Omega_{p}\right) .
$$

Since the basis elements $\omega_{k}^{p}$ form a basis, we have that $\sum a_{k} \omega_{k}^{p}=0$ is equivalent to $a_{k}=0$, therefore we can write it in a purely topological fashion (independent of metric and material properties)

$$
\sum_{k=1, j=0}^{p^{2}, 2 p(p+1)-1} \mathrm{E}_{k, j}^{2,1} h_{p, j}=\sum_{k=1}^{p^{2}} J_{k} .
$$

In matrix notation, this equation takes the more compact form

$$
\mathbf{E}^{2,1} \boldsymbol{h}_{p}=\boldsymbol{J}
$$

where $\boldsymbol{h}_{p}$ denotes the row vector with $j$-th entry equal to $h_{p, j}$ and $\boldsymbol{J}$ represents the row vector with $j$-th entry equal to $J_{j}$.

The key factor that allows the construction of a purely topological law at the discrete level, (49), is the appropriate definition of a set of basis functions such that (42) and (44) are satisfied.

\subsubsection{The discrete system of equations}

Having defined a natural inner product, (19), having constructed a set of basis functions with which to discretize the unknown physical quantities $\psi$ and $\vec{h}_{p}$, (32) and (35), and having derived the equivalent discrete topological law, (49), we can now revisit the weak formulation of the Poisson problem, (22).

Consider the computational domain $\Omega_{p}$ in the $(r, z)$-plane and its tessellation $\mathcal{T}\left(\Omega_{p}\right)$ consisting of $N$ arbitrary quadrilaterals (possibly curved), $\Omega_{m}$ with $m=1, \ldots, N$. We assume that all quadrilateral elements $\Omega_{m}$ can be obtained from a map $\Phi_{m}:(\xi, \eta) \in Q \rightarrow(r, z) \in \Omega_{m}$. Then the pushforward $\Phi_{m, *}$ maps functions in the reference element $Q$ to functions in the physical element $\Omega_{m}$, see for example [1,21]. For this reason it suffices to explore the analysis on the reference domain $Q$.

Remark 1. If a differential geometry formulation was used, the physical quantities would be represented by differential $k$-forms and the map $\Phi_{m}:(\xi, \eta) \in Q \rightarrow(r, z) \in \Omega_{m}$ would generate a pullback, $\Phi_{m}^{*}$, mapping $k$-forms in physical space, $\Omega_{m}$, to $k$-forms in the reference element, $Q$.

Let $U$ and $V$ be finite-dimensional polynomial spaces such that $U=\operatorname{span}\left\{\omega_{1}^{p}, \ldots, \omega_{p^{2}}^{p}\right\} \subset L^{2}(Q)$ and $V=\operatorname{span}\left\{\vec{\epsilon}_{0}^{p}, \ldots\right.$, $\left.\vec{\epsilon}_{2 p(p+1)-1}^{p}\right\} \subset H(\nabla \times, Q)$. The mimetic formulation for the discrete solution of (14) on $Q$ is given by

$$
\left\{\begin{array}{l}
\text { Find } \psi_{h} \in U \text { and } \vec{h}_{p, h} \in V \text { such that: } \\
\left\langle\psi_{h}, \nabla \times \vec{\epsilon}_{j}^{p}\right\rangle_{Q}=\left\langle\vec{h}_{p, h}, \vec{\epsilon}_{j}^{p}\right\rangle_{\mathbb{K}, Q}, \quad j=0, \ldots, 2 p(p+1)-1, \\
\left\langle\nabla \times \vec{h}_{p}, \omega_{j}^{p}\right\rangle_{Q}=\left\langle J, \omega_{j}^{p}\right\rangle_{Q}, \quad j=1, \ldots, p^{2} .
\end{array}\right.
$$

Well-posedness of this discrete formulation follows directly from the fact that $V \subset H(\nabla \times, Q), U \subset L^{2}(Q)$ and $\mathcal{Z}_{h}:=$ $\left\{\vec{v}_{h} \in V \mid \nabla \times \vec{v}_{h}\right\} \subset \mathcal{Z}:=\{\vec{v} \in H(\nabla \times, Q) \mid \nabla \times \vec{v}\}$. For a more detailed discussion see [56].

Substituting $\psi_{h}, \vec{h}_{p, h}$ by their expressions and $J$ by its expansion, as in (39), (34) and (39) respectively, and using both the linearity of the inner product and (42) we obtain 


$$
\left\{\begin{array}{l}
\text { Find } \psi_{h} \in U \text { and } \vec{h}_{p, h} \in V \text { such that: } \\
\sum_{i=1, k=1}^{p^{2}} \mathrm{E}_{k, j}^{2,1} \psi_{i}\left\langle\omega_{i}^{p}, \omega_{k}^{p}\right\rangle_{Q}=\sum_{k=0}^{2 p(p+1)-1} h_{p, k}\left\langle\vec{\epsilon}_{k}^{p}, \vec{\epsilon}_{j}^{p}\right\rangle_{\mathbb{K}, Q}, \quad j=0, \ldots, 2 p(p+1)-1, \\
\sum_{i=0, k=1}^{2 p(p+1)-1, p^{2}} \mathrm{E}_{k, i}^{2,1} h_{p, i}\left\langle\omega_{k}^{p}, \omega_{j}^{p}\right\rangle_{Q}=\sum_{k=1}^{p^{2}} J_{k}\left\langle\omega_{k}^{p}, \omega_{j}^{p}\right\rangle_{Q}, \quad j=1, \ldots, p^{2} .
\end{array}\right.
$$

Note that we have used $\nabla \times \vec{\epsilon}_{j}^{p}=\sum_{k=1}^{p^{2}} \mathrm{E}_{k, j} \omega_{k}^{p}$, a special case of (42). This formulation takes a more compact form in matrix notation

$$
\left\{\begin{array}{l}
\text { Find } \boldsymbol{\psi} \in \mathbb{R}^{p^{2}} \text { and } \boldsymbol{h}_{p} \in \mathbb{R}^{2 p(p+1)} \text { such that: } \\
\left(\mathbf{E}^{2,1}\right)^{\top} \mathbf{N} \boldsymbol{\psi}=\mathbf{M} \boldsymbol{h}_{p} \\
\mathbf{N E}^{2,1} \boldsymbol{h}_{p}=\mathbf{N} \mathbf{J}
\end{array}\right.
$$

with

$$
\mathrm{N}_{i, j}:=\left\langle\omega_{i}^{p}, \omega_{j}^{p}\right\rangle_{Q} \quad \text { and } \quad \mathrm{M}_{i, j}:=\left\langle\vec{\epsilon}_{i}^{p}, \vec{\epsilon}_{j}^{p}\right\rangle_{\mathbb{K}, Q} .
$$

As expected, for homogeneous Dirichlet boundary conditions, $\psi_{b}=0$, the system of algebraic equations is symmetric.

\subsection{Grad-Shafranov problem and its discrete solution}

The discrete solution of the Grad-Shafranov problem (4) can be directly obtained combining (53) with the iterative procedures described in Section 2.2.1 (non-eigenvalue case) and Section 2.2.2 (eigenvalue case).

\subsubsection{Non-eigenvalue case}

For the non-eigenvalue case we replace in (6) the continuous operators by their discrete versions (53). Hence, for each iteration $k$ the following algebraic system of equations is solved

$$
\left\{\begin{array}{l}
\text { Find } \boldsymbol{\psi}^{k+1} \in \mathbb{R}^{p^{2}} \text { and } \boldsymbol{h}_{p}^{k+1} \in \mathbb{R}^{2 p(p+1)} \text { such that: } \\
\left(\mathbf{E}^{2,1}\right)^{\top} \mathbf{N} \psi^{k+1}=\mathbf{M} \boldsymbol{h}_{p}^{k+1} \\
\mathbf{N E}^{2,1} \boldsymbol{h}_{p}^{k+1}=\mathbf{N} \mathbf{J}^{k}
\end{array}\right.
$$

with

$$
J_{n}^{k}=\int_{\xi_{i-1}}^{\xi_{i}} \int_{\eta_{j-1}}^{\eta_{j}} J_{\phi}\left(\psi_{h}^{k}(r, z), r, z\right) \mathrm{d} \xi \mathrm{d} \eta, \quad n=j+(i-1) p, \quad i, j=1, \ldots, p .
$$

As before, the iterative procedure is stopped once the residual between two consecutive iterations satisfies

$$
\left\|\nabla \times \vec{h}_{p, h}^{k+1}-J_{h}^{k+1}\right\|_{L^{s}}<\epsilon,
$$

with $\epsilon \ll 1$ and $s \in \mathbb{N}$.

\subsubsection{Eigenvalue case}

In a similar way, for the eigenvalue case we just replace in (11) and (12) the continuous operators by their discrete versions (53). Therefore, for each iteration $k$ a new eigen-pair $\left(\bar{\psi}^{k+1}, \sigma^{k+1}\right)$ is computed by solving the algebraic system of equations

$$
\left\{\begin{array}{l}
\text { Find } \boldsymbol{\psi}^{k+1} \in \mathbb{R}^{p^{2}} \text { and } \boldsymbol{h}_{p}^{k+1} \in \mathbb{R}^{2 p(p+1)} \text { such that: } \\
\left(\mathbf{E}^{2,1}\right)^{\top} \mathbf{N} \boldsymbol{\psi}^{k+1}=\mathbf{M} \boldsymbol{h}_{p}^{k+1} \\
\mathbf{N E}^{2,1} \boldsymbol{h}_{p}^{k+1}=\sigma^{k} \mathbf{N} \boldsymbol{J}^{k}
\end{array}\right.
$$

and then normalizing

$$
\bar{\psi}^{k+1}=\frac{\psi^{k+1}}{\left\|\psi_{h}^{k+1}(\xi, \eta)\right\|_{L^{s}}} \quad \text { and } \quad \sigma^{k+1}=\frac{\sigma^{k}}{\left\|\psi_{h}^{k+1}(\xi, \eta)\right\|_{L^{s}}},
$$


with

$$
J_{n}^{k}=\int_{\xi_{i-1}}^{\xi_{i}} \int_{\eta_{j-1}}^{\eta_{j}} J_{\phi}\left(\bar{\psi}_{h}^{k}(r, z), r, z\right) \mathrm{d} \xi \mathrm{d} \eta, \quad n=j+(i-1) p, \quad i, j=1, \ldots, p .
$$

As before, the iterative procedure is stopped once the residual satisfies (56).

\section{Numerical test cases}

In order to assess the accuracy and robustness of the proposed Grad-Shafranov solver we apply it to the solution of different test cases. We start by analyzing the convergence properties of the solver on different grids by solving equilibria where exact solutions are known, Section 3.1. We then proceed to address the case where the computational domain coincides with the plasma domain, Section 3.2. We first present results for the case of smooth plasma boundaries for both linear, Section 3.2.2, and non-linear eigenvalue problems, Section 3.2.3. We finalize with an application to an X-point configuration, Section 3.3.

The choice of the specific test cases addressed here was made with the objective of comparing the proposed solver with results from recently published high order solvers, namely $[35,46,59]$. Therefore most of the test cases discussed here are also presented either in [35] or [59]. A summary of the different profile models used in the test cases is presented in Table 1.

Table 1

A summary of the profile models used in the test cases.

\begin{tabular}{lll}
\hline Equilibria & $f$ model & $P$ model \\
\hline Soloviev & $f(\psi)=f_{0}$ & $P(\psi)=C \psi+P_{0}$ \\
FRC & $f(\psi)=0$ & $P(\psi)=P_{0}+P_{1} \frac{\psi^{2}}{\psi_{0}^{2}}$ \\
Spheromak & $f(\psi)=f_{0} \psi$ & $P(\psi)=P_{0} \frac{\psi}{\psi_{0}}$ \\
Linear eigenvalue & $f(\psi)=0$ & $P(\psi)=\frac{C_{1}+C_{2} r^{2}}{2 \mu_{0}} \psi^{2}+P_{0}$ \\
non-linear eigenvalue & $f(\psi)=0$ & $P(\psi)=\frac{C_{1}+C_{2} \psi^{2}}{\mu_{0}}\left(1-e^{-\frac{\psi^{2}}{\eta}}\right)$ \\
X-point (Soloviev) & $f(\psi)=\sqrt{2 \mu_{0} A \psi}$ & $P(\psi)=(1-A) \psi$ \\
X-point (linear eigenvalue) & $f(\psi)=0$ & $P(\psi)=\frac{C_{1}+C_{2} r^{2}}{2 \mu_{0}} \psi^{2}+P_{0}$ \\
X-point (non-linear eigenvalue) & $f(\psi)=0$ & $P(\psi)=\frac{C_{1}+C_{2} \psi^{2}}{\mu_{0}}\left(1-e^{-\frac{\psi^{2}}{\eta}}\right)$ \\
\hline
\end{tabular}

\subsection{Convergence tests}

The convergence tests focus mainly on both $h$ - (mesh refinement) and $p$ - (increase of polynomial degree of basis functions) convergence studies. We intend to show optimal convergence rates of order $p+1$ for $h$-refinement and exponential convergence rates for $p$-refinement. Another important aspect we intend to show is the robustness of the present method with respect to highly curved meshes. For this reason we will compare the convergence rates on orthogonal meshes with the ones on highly curved meshes. We will use three plasma configurations where exact solutions are known: (i) Soloviev solution in Section 3.1.1, (ii) field-reversed configuration (FRC) in Section 3.1.2 and (iii) spheromak configuration in Section 3.1.3.

\subsubsection{Soloviev test case}

The first test case corresponds to a Soloviev solution of the Grad-Shafranov equation. This solution is one of the most widely used analytical solutions of the Grad-Shafranov equation. This special case corresponds to a constant model $f(\psi)=f_{0}$ combined with a linear model $P(\psi)=C \psi+P_{0}$. This leads to the following Grad-Shafranov equation

$$
\begin{cases}\nabla \times(\mathbb{K} \nabla \times \psi)=\frac{r}{\mu_{0}} & \text { in } \quad \Omega_{p}, \\ \psi=\psi_{a} & \text { on } \partial \Omega_{p},\end{cases}
$$

where we have set $C=\frac{1}{\mu_{0}}$. Note that since we are solving the Grad-Shafranov equation on a rectangular domain, here $\Omega_{p}$ stands for a rectangular domain, which includes the plasma.

We outline here the methodology presented in $[15,59]$ to obtain physically relevant solutions to this equation. The analytical solution, $\psi_{a}(r, z)$, is constructed by adding to the particular solution $\frac{r^{4}}{8}$ a linear combination of three homogeneous solutions,

$$
\psi_{a}(r, z)=\frac{r^{4}}{8}+d_{1}+d_{2} r^{2}+d_{3}\left(r^{4}-4 r^{2} z^{2}\right) .
$$



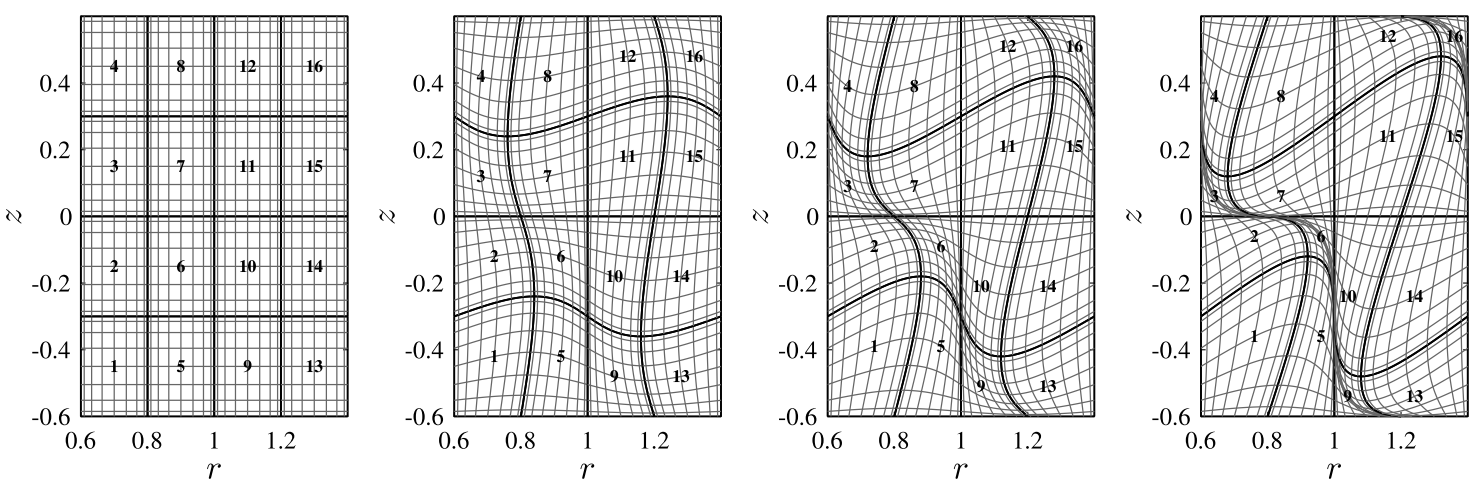

Fig. 4. Example of mesh deformation generated by (64) for $c=0.0,0.1,0.2,0.3$ used in the ITER-like case. Mesh corresponding to $4 \times 4$ elements of polynomial degree $p=8$.
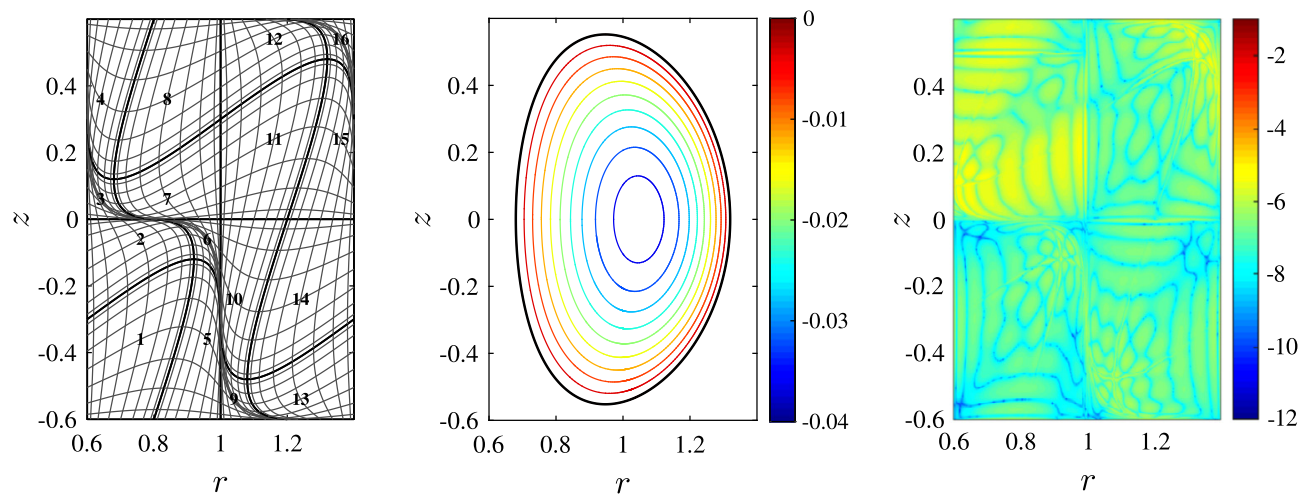

Fig. 5. Numerical solution of the Soloviev test case, (60), for ITER parameters, $\epsilon=0.32, \kappa=1.7, \delta=0.33$. From left to right: (i) computational mesh, with curvature parameter $c=0.3,4 \times 4$ elements of polynomial degree $p=8$, (ii) numerical solution using the mesh in (i), $\psi_{h}$, and (iii) logarithmic error between the analytical solution and the numerical one, $\log _{10}\left|\psi_{a}-\psi_{h}\right|$.

The coefficients $d_{i}$ are determined by imposing a physically relevant shape to the contour line $\psi_{a}(r, z)=0$, the plasma cross section. The shape of the plasma is parameterized by three variables, see [15,59] for a detailed discussion: inverse aspect-ratio $\epsilon$, elongation $\kappa$, and triangularity $\delta$. The imposed boundary conditions correspond to the following equations

$$
\begin{cases}\psi_{a}(1 \pm \epsilon, 0)=0 & \text { Lateral shape conditions, } \\ \psi_{a}(1-\delta \epsilon, \kappa \epsilon)=0 & \text { Vertical shape condition, }\end{cases}
$$

which can be expressed as

$$
\left(\begin{array}{ccc}
1 & (1+\epsilon)^{2} & (1+\epsilon)^{4} \\
1 & (1-\epsilon)^{2} & (1-\epsilon)^{4} \\
1 & (1-\delta \epsilon)^{2} & (1-\delta \epsilon)^{4}-4(1-\delta \epsilon)^{2} \kappa^{2} \epsilon^{2}
\end{array}\right)\left(\begin{array}{l}
d_{1} \\
d_{2} \\
d_{3}
\end{array}\right)=-\frac{1}{8}\left(\begin{array}{c}
(1+\epsilon)^{4} \\
(1-\epsilon)^{4} \\
(1-\delta \epsilon)^{4}
\end{array}\right) .
$$

The numerical method proposed in this work is applied to the solution of two Soloviev solution cases, as in [59]. One corresponds to an ITER-like cross section where $\epsilon=0.32, \kappa=1.7$ and $\delta=0.33$. The other solution corresponds to an NTSX-like cross section where $\epsilon=0.78, \kappa=2.0$ and $\delta=0.35$.

For this test case we consider meshes obtained by the transformation of the unit square $[-1,1]^{2}$ to curvilinear coordinates on the domain $\left[r_{-}, r_{+}\right] \times\left[z_{-}, z_{+}\right]$by the mapping given as $(r, z)=\Phi(\xi, \eta)$, with

$$
\left\{\begin{array}{l}
r(\xi, \eta)=(\xi+c \sin (\pi \xi) \sin (\pi \eta)+1) \frac{r_{+}-r_{-}}{2}, \\
z(\xi, \eta)=(\eta+c \sin (\pi \xi) \sin (\pi \eta)+1) \frac{z_{+}-z_{-}}{2} .
\end{array}\right.
$$

Fig. 4 shows the meshes generated by (64) with increasing values of $c$, for the solution domain used in the ITER-like case.

The mimetic spectral element method is capable of very accurately reproducing the analytical solution for both the ITER and the NSTX cases even on highly deformed meshes, see Fig. 5 and Fig. 6 respectively. 

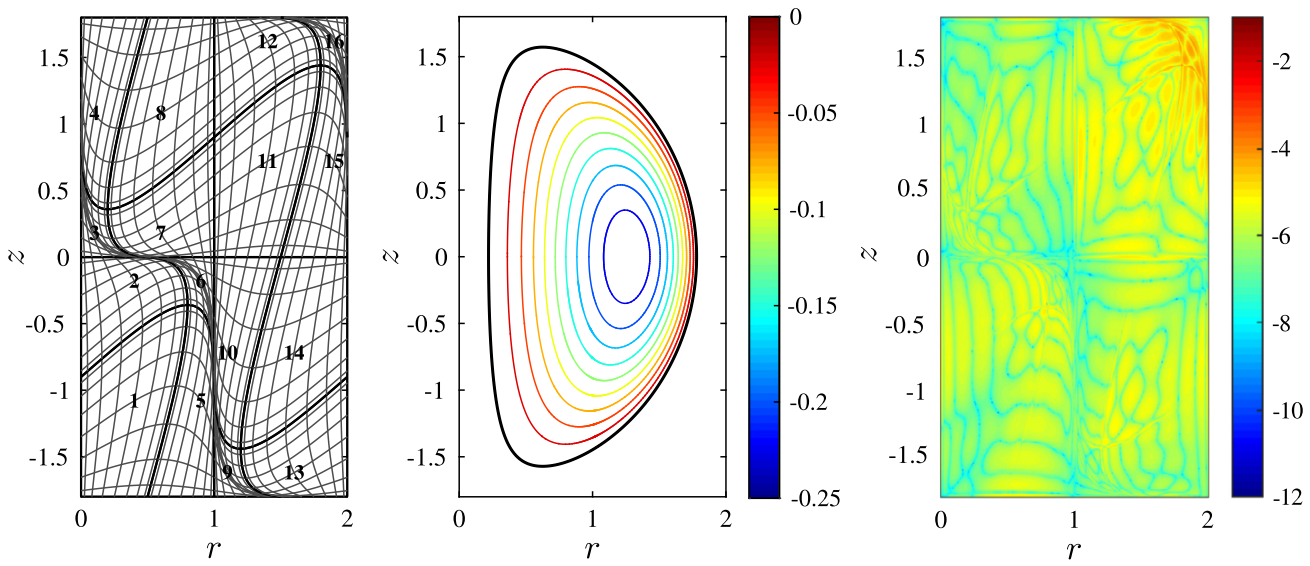

Fig. 6. Numerical solution of the Soloviev test case, (60), for NSTX parameters, $\epsilon=0.78, \kappa=2.0, \delta=0.35$. From left to right: (i) computational mesh, with curvature parameter $c=0.3,4 \times 4$ elements of polynomial degree $p=8$, (ii) numerical solution using the mesh in (i), $\psi_{h}$, and (iii) logarithmic error between the analytical solution and the numerical one, $\log _{10}\left|\psi_{a}-\psi_{h}\right|$.
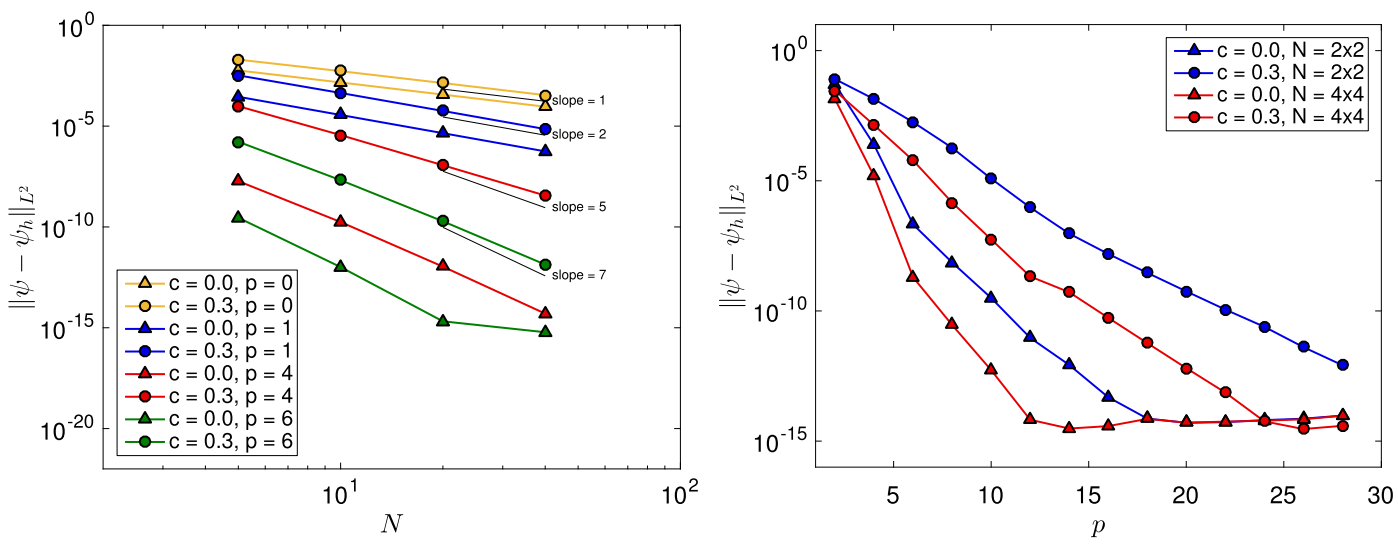

Fig. 7. Convergence plots for the numerical solution of the Soloviev test case (60) for ITER parameters, $\epsilon=0.32, \kappa=1.7, \delta=0.33$. Left: $h$-convergence plots. Right: $p$-convergence plots. Convergence plots computed for meshes with deformation, $c=0.0$ and $c=0.3$.

The convergence tests for $h$-refinement and $p$-refinement show very good convergence rates. For $h$-convergence, Fig. 7 left, we can see that the mimetic spectral element solver preserves high convergence rates very close to $p+1$ even on highly deformed meshes. Regarding p-convergence, Fig. 7 right, we observe the same robustness of the method, with convergence rates maintaining their exponential character on highly deformed meshes. Furthermore, it is possible to observe convergence to machine accuracy.

\subsubsection{FRC test case}

The second test case corresponds to a field-reversed configuration (FRC). This solution is characterized by zero toroidal field $f(\psi)=0$ combined with a quadratic model for pressure $P(\psi)=P_{0}+P_{1} \frac{\psi^{2}}{\psi_{0}^{2}}$, where $P_{0}$ and $P_{1}$ are free constants and $\psi_{0}$ is the flux at the magnetic axis, also a free parameter. This corresponds to the following Grad-Shafranov equation

$$
\begin{cases}\nabla \times(\mathbb{K} \nabla \times \psi)=2 r P_{1} \frac{\psi}{\psi_{0}^{2}} & \text { in } \Omega_{p}, \\ \psi=0 & \text { on } \partial \Omega_{p},\end{cases}
$$

where $\Omega_{p}=\left[0, L_{r}\right] \times\left[0, L_{z}\right]$.

An extensive derivation of the analytical solution under these conditions is presented in [58]. Here we outline this procedure. The Grad-Shafranov equation can be written in terms of dimensionless variables $\bar{r}=\frac{r}{L_{r}}$ and $\bar{z}=\frac{z}{L_{z}}$ and the analytical solution obtained by the method of separation of variables such that

$$
\psi_{a}(\bar{r}, \bar{z})=W(\bar{r}) T(\bar{z}) .
$$

A solution for $T(\bar{z})$ that satisfies the boundary conditions is given by $T(\bar{z})=\sin (\pi \bar{z})$. Introducing the change of variables $\zeta=\frac{\mathbf{i} L_{r}^{2}}{\psi_{0}} \sqrt{2 \mu_{0} P_{1}} \bar{r}^{2}$ the equation for $W(\bar{z})$ becomes 

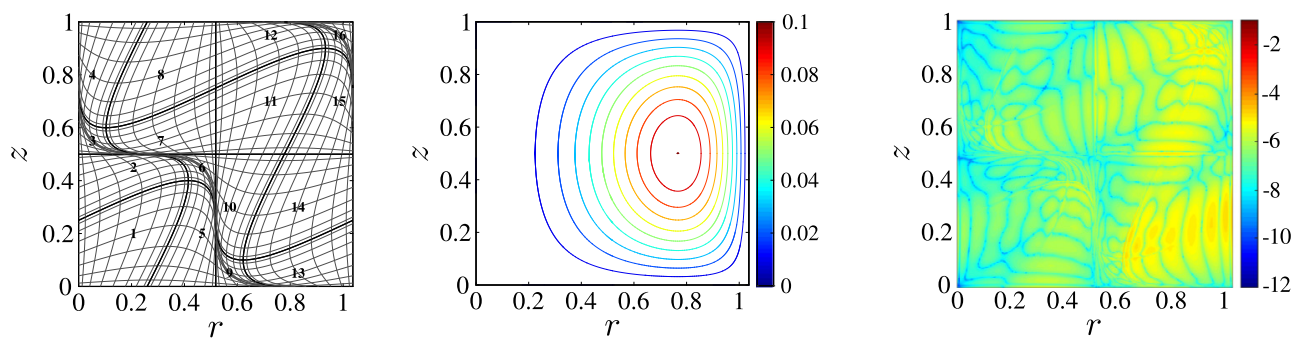

Fig. 8. Numerical solution of the FRC test case, (65), with $\psi_{0}=0.1, L_{z}=1.0, \mu_{0} P_{1}=0.277, r_{0}=0.7670524200738164$ and $L_{r}=1.0367471722606991$. From left to right: (i) computational mesh, with curvature parameter $c=0.3,4 \times 4$ elements of polynomial degree $p=8$, (ii) numerical solution using the mesh in (i), $\psi_{h}$, and (iii) logarithmic error between the analytical solution and the numerical one, $\log _{10}\left|\psi_{a}-\psi_{h}\right|$.
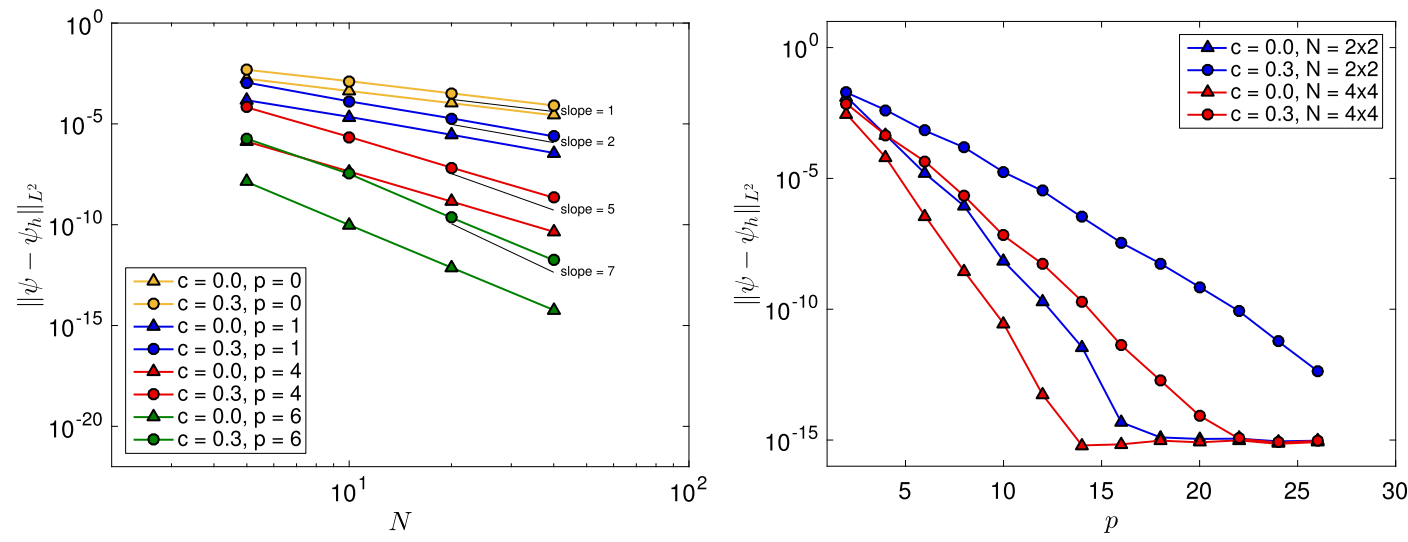

Fig. 9. Convergence plots for the numerical solution of the FRC test case (65), with $\psi_{0}=0.1, L_{z}=1.0, \mu_{0} P_{1}=0.277, r_{0}=0.7670524200738164$ and $L_{r}=1.0367471722606991$. Left: $h$-convergence plots. Right: $p$-convergence plots. Convergence plots computed for meshes with deformation, $c=0.0$ and $c=0.3$.

$$
\frac{\mathrm{d}^{2} W}{\mathrm{~d} \zeta^{2}}+\left(\frac{\mathbf{i} k}{\zeta}-\frac{1}{4}\right) W=0,
$$

with $k=\frac{\pi^{2}}{4 L_{z}^{2} \sqrt{d}}, d=\frac{2 \mu_{0} P_{1}}{\psi_{0}^{2}}$ and $\mathbf{i}=\sqrt{-1}$. Equation (67) is Whittaker's equation with $m=\frac{1}{2}$. The solution that is real-valued and zero on the $z$-axis $(\zeta=0)$ is given by $W(\zeta)=-\mathbf{i} C M_{\mathbf{i} k, \frac{1}{2}}(\zeta)$, with $C$ a real number and $M_{i k, \frac{1}{2}}(\zeta)$ Whittaker's $M$ function. By imposing the radial boundary condition, $W\left(\mathbf{i} \sqrt{d} \bar{r}^{2}\right)=0$, the lowest physically realistic value for $L_{r}$ is determined by finding the first non-zero root of $-\mathbf{i} M_{\mathbf{i} k, \frac{1}{2}}(\zeta), \zeta_{1}$. The location of the magnetic O-point on the midplane $\zeta=\zeta_{0}$ corresponds to the maximum of $-\mathbf{i} M_{\mathbf{i} k, \frac{1}{2}}(\zeta)$ in the interval $\left[0, \zeta_{1}\right]$. The analytical solution is then given by

$$
\psi_{a}(r, z)=\psi_{0} \frac{M_{\mathbf{i} k, \frac{1}{2}}\left(\mathbf{i} \sqrt{d} r^{2}\right)}{M_{\mathbf{i} k, \frac{1}{2}}\left(\mathbf{i} \sqrt{d} r_{0}^{2}\right)} \sin \left(\frac{\pi}{L_{z} z}\right),
$$

with $r_{0}=\zeta_{0}^{\frac{1}{2}} d^{-\frac{1}{4}}$ and $L_{r}=\zeta_{1}^{\frac{1}{2}} d^{-\frac{1}{4}}$.

The mimetic spectral element method is applied to the FRC solution with parameters $\psi_{0}=0.1, L_{z}=1.0, \mu_{0} P_{1}=0.277$, $r_{0}=0.7670524200738164$ and $L_{r}=1.0367471722606991$, as in [35]. As in the Soloviev test case, Section 3.1.1, we use the same mapping to generate the mesh deformation, (64), on the domain $\left[0, L_{r}\right] \times\left[0, L_{z}\right]$.

We see that the proposed method accurately computes the FRC solution, showing small errors even on a highly deformed mesh, see Fig. 8.

The convergence tests for the FRC test case confirm the results obtained for the Soloviev solution. We can observe high $h$-convergence rates very close to order $p+1$, Fig. 9 left. Similar behavior can be seen for $p$-convergence with the method converging exponentially fast for both straight and curved meshes, Fig. 9 right. Also for this test case machine accuracy is achieved.

As pointed out in [58], FRC equilibria are often very elongated. In order to study the dependence of the error in the numerical solution with respect to the plasma elongation $\frac{L_{z}}{L_{r}}$, we have computed the error as a function of the elongation for a mesh with $20 \times 20$ elements of polynomial degree $p=1$ (Fig. 10 left) and for a mesh with $4 \times 4$ elements of polynomial degree $p=5$ (Fig. 10 right). As can be seen, the error increases slowly with the elongation (blue line). Since the area of the plasma increases with the elongation, a better comparison is the error normalized by the area of the plasma (red line). The 

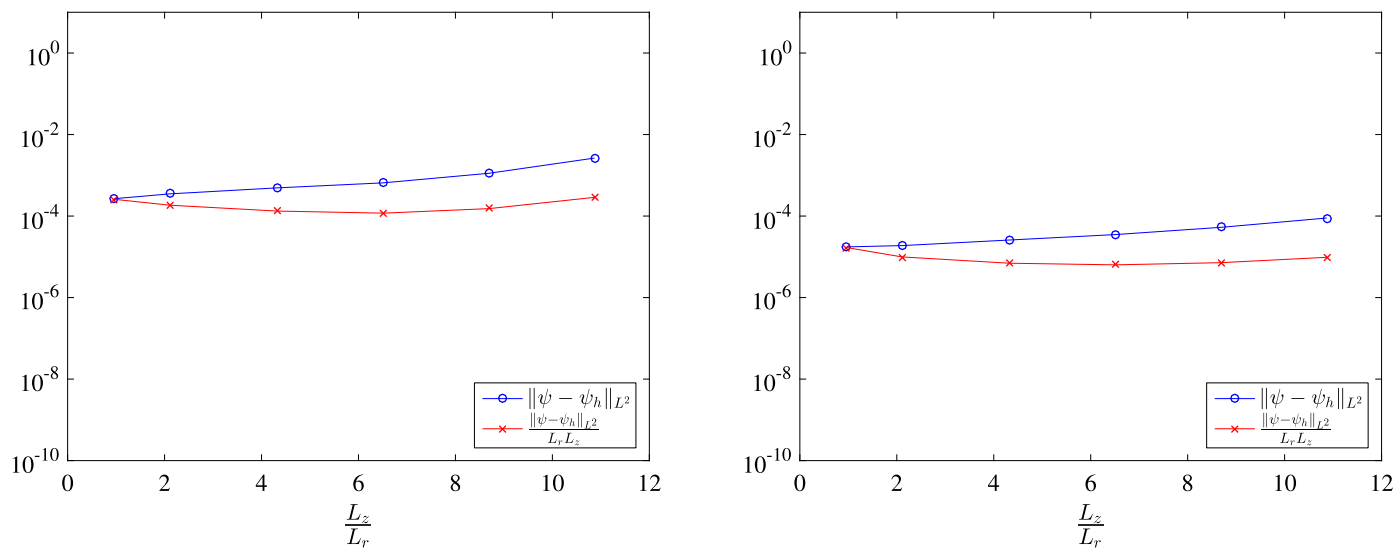

Fig. 10. Error for the numerical solution of the FRC test case (65), with $\psi_{0}=0.1, L_{z}=[1.0,2.0,4.0,6.0,8.0,10.0]$ and $\mu_{0} P_{1}=0.277$. Left: mesh with $20 \times 20$ elements of polynomial degree $p=1$. Right: mesh with $4 \times 4$ elements of polynomial degree $p=5$. (For interpretation of the references to color in this figure, the reader is referred to the web version of this article.)
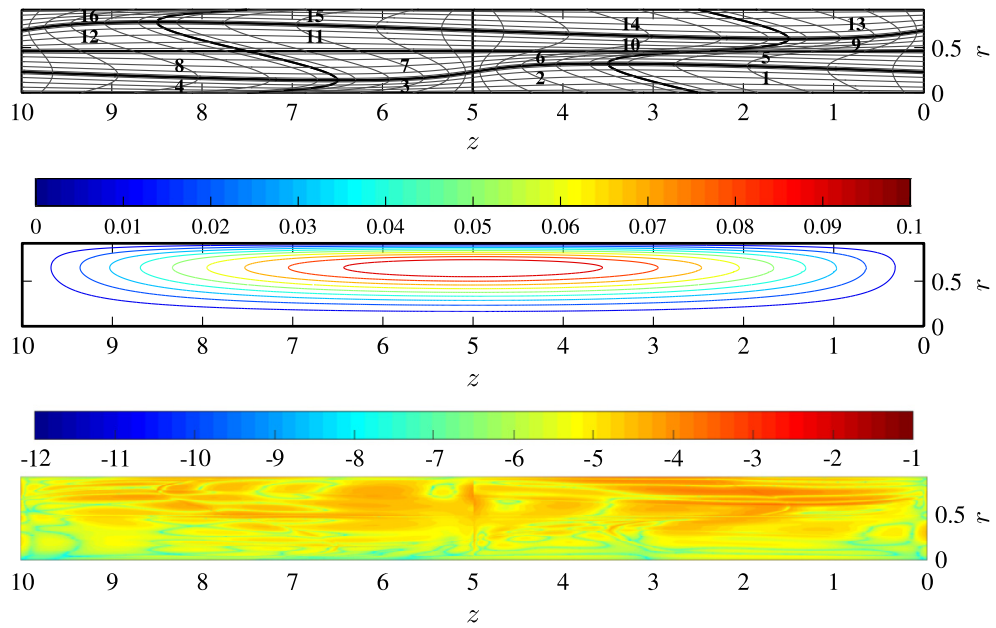

Fig. 11. Numerical solution of the FRC test case, (65), with $\psi_{0}=0.1, L_{z}=10.0, \mu_{0} P_{1}=0.277, r_{0}=0.6508076929394377$ and $L_{r}=0.9199638879139966$. From top to bottom: (i) computational mesh, with curvature parameter $c=0.2,4 \times 4$ elements of polynomial degree $p=5$, (ii) numerical solution using the mesh in (i), $\psi_{h}$, and (iii) logarithmic error between the analytical solution and the numerical one, $\log _{10}\left|\psi_{a}-\psi_{h}\right|$.

normalized error shows a very small variation with the elongation of the plasma. As an example, we present in Fig. 11 the numerical solution for a plasma with elongation 10.87 .

\subsubsection{Spheromak test case}

The third test case used to determine the $h$ - and $p$-convergence properties of the proposed method is the cylindrical spheromak configuration. This case is obtained by a linear toroidal field $f(\psi)=f_{0} \psi$ together with a linear pressure profile $P(\psi)=P_{0} \frac{\psi}{\psi_{0}}$, where $f_{0}$ and $P_{0}$ are constants and $\psi_{0}$ is the flux at the magnetic axis, also a free parameter. With these models the Grad-Shafranov problem becomes

$$
\begin{cases}\nabla \times(\mathbb{K} \nabla \times \psi)=\frac{P_{0} r}{\psi_{0}}-\frac{f_{0}^{2} \psi}{\mu_{0} r} & \text { in } \Omega_{p}, \\ \psi=0 & \text { on } \partial \Omega_{p},\end{cases}
$$

where $\Omega_{p}=\left[0, L_{r}\right] \times\left[0, L_{z}\right]$.

Here we simply outline the derivation of the analytical solution as presented in [4]. The methodology to obtain the analytical solution is very similar to the one used in the FRC case, Section 3.1.2. The first step is to multiply (69) by $r$ and rewrite (69) in terms of the normalized quantities $\bar{\psi}=\frac{\psi}{\psi_{0}}, \bar{r}=\frac{r}{r_{0}}, \bar{z}=\frac{z}{r_{0}}, \bar{f}_{0}=f_{0} r_{0}, \bar{k}=k r_{0}=r_{0} \frac{\pi}{L_{z}}$ and with respect to $\chi=\bar{\psi}+\beta \bar{r}^{2} \bar{f}_{0}^{-2}$, where $\beta=\pi^{2} \mu_{0} P_{0} r_{0}^{4} \psi_{0}^{-2}$ and $r_{0}$ is the radial coordinate of the plasma's axis:

$$
\bar{r} \frac{\partial}{\partial \bar{r}}\left(\frac{1}{\bar{r}} \frac{\partial \chi}{\partial \bar{r}}\right)+\frac{\partial^{2} \chi}{\partial \bar{z}}+\bar{f}_{0} \chi=0 \text {. }
$$



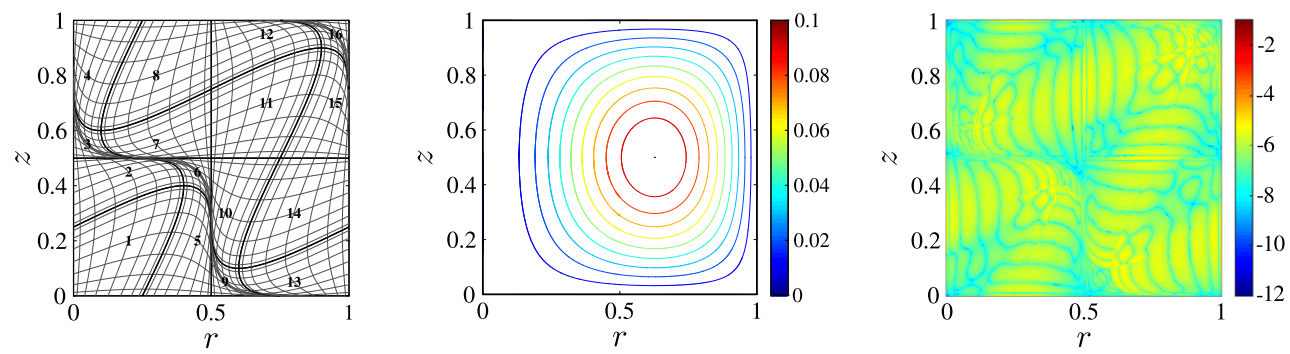

Fig. 12. Numerical solution of the spheromak test case, (69), with $\psi_{0}=0.1, L_{r}=1.0, L_{z}=1.0$ and $f_{0}=4.954954595474438$. From left to right: (i) computational mesh, with curvature parameter $c=0.3,4 \times 4$ elements of polynomial degree $p=8$, (ii) numerical solution using the mesh in (i), $\psi_{h}$, and (iii) logarithmic error between the analytical solution and the numerical one, $\log _{10}\left|\psi_{a}-\psi_{h}\right|$.
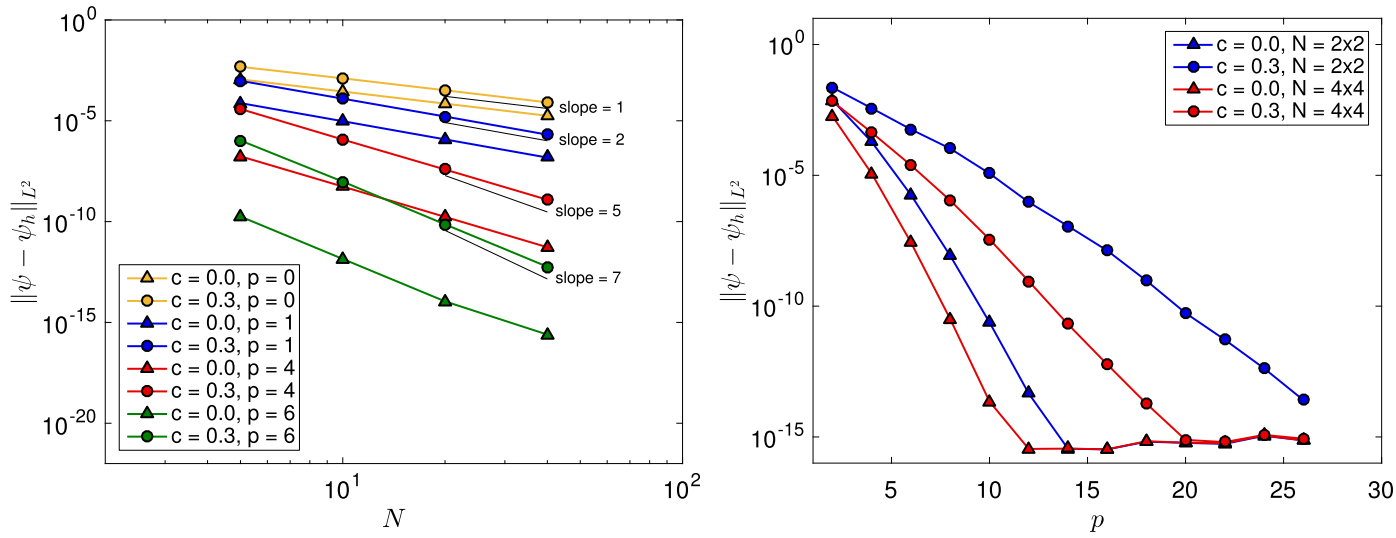

Fig. 13. Convergence plots for the numerical solution of the spheromak test case (65), with $\psi_{0}=0.1, L_{r}=1.0, L_{z}=1.0$ and $f_{0}=4.954954595474438$. Left: $h$-convergence plots. Right: $p$-convergence plots. Convergence plots computed for meshes with deformation, $c=0.0, c=0.1, c=0.2$ and $c=0.3$.

The solution can now be obtained by the method of separation of variables such that

$$
\chi(\bar{r}, \bar{z})=\bar{r} g(\bar{r}) \sin (\bar{k} \bar{z}) .
$$

Introducing the change of variables $x^{2}=\left(\bar{f}_{0}{ }^{2}-\bar{k}^{2}\right) \bar{r}^{2}$ the equation for $g(x)$ becomes:

$$
x^{2} \frac{\mathrm{d}^{2} g}{\mathrm{~d} x^{2}}+x \frac{\mathrm{d} g}{\mathrm{~d} x}+\left(x^{2}-1\right) g=0,
$$

which is Bessel's equation with $n=1$. The physically relevant solution is then

$$
\psi_{a}(r, z)=\psi_{0} \frac{\chi_{11}}{\chi_{01} L_{r}} \frac{B_{1}\left(\frac{\chi_{11} r}{L_{r}}\right)}{B_{1}\left(\chi_{01}\right)} r \sin \left(\frac{\pi r}{L_{z}}\right)-\frac{\pi^{2} \mu_{0} P_{0} r}{f_{0} \psi_{0}^{2}},
$$

with $B_{n}$ the $n$-th Bessel function of the first kind, $\chi_{i j}$ the $j$-th positive zero of Bessel function $B_{i}$. The constant $f_{0}$ is then given by $f_{0}=\sqrt{\chi_{11}^{2} L_{r}^{-2}+k^{2}}$.

For this test case we apply the numerical method developed in this article to the spheromak solution with parameters $\psi_{0}=0.1, L_{r}=1.0, L_{z}=1.0, P_{0}=0.0$ and $f_{0}=4.954954595474438$, as in [35].

As can be seen in Fig. 12, the mimetic spectral element method is capable of accurately reproducing the results on a highly curved mesh obtained with the mapping (64), on the domain $\left[0, L_{r}\right] \times\left[0, L_{z}\right]$. The convergence tests for the spheromak test case confirm the results obtained previously. We can observe high $h$-convergence rates close to order $p+1$, Fig. 13 left. Similar behavior can be seen for $p$-convergence with the method converging exponentially fast for both straight and curved meshes, Fig. 13 right. Machine accuracy is also achieved in this test case.

\subsection{Curved plasma boundary}

In this section we assess the ability of the proposed numerical model to solve fixed boundary problems where the prescribed plasma boundary is curved. For this purpose the method is tested first on the Soloviev solutions presented in Section 3.1.1 (now with curved plasma boundary), then on both linear and non-linear eigenvalue problems, and finally we test it on a plasma with an X-point. 
In all cases, the plasma boundary is prescribed parametrically such that $r_{b}=\gamma_{r}(s)$ and $z_{b}=\gamma_{z}(s)$ with $s \in\left[s_{-}, s_{+}\right]$. The computational mesh is prescribed using a transfinite mapping, see [27].

\subsubsection{Soloviev test case}

In order to assess the accuracy of the proposed method for the solution of a curved boundary plasma shape, we first apply it to the Soloviev problem introduced in Section 3.1.1. For this case the plasma boundary is given by the equation

$$
\frac{r^{4}}{8}+d_{1}+d_{2} r^{2}+d_{3}\left(r^{4}-4 r^{2} z^{2}\right)=0
$$

with $d_{1}, d_{2}, d_{3}$ as in (63).

In Fig. 14 an example solution for ITER parameters is presented and in Fig. 15 another example solution for NSTX parameters is shown. As can be seen, both solutions are well reconstructed by the proposed method. The NSTX case has larger errors due to the more deformed underlying mesh. As was seen in Section 3.1.1, a higher mesh deformation leads to higher errors.

The NSTX case convergence tests for $h$-refinement and $p$-refinement show very good convergence rates both for $\psi_{h}$ and for $\vec{h}_{h}$. For $h$-convergence, Fig. 16 left and Fig. 17 left, we can see that the mimetic spectral element solver preserves high convergence rates of $p+1$. Regarding $p$-convergence, Fig. 16 right and Fig. 17 right, we observe the same robustness of the method, with convergence rates maintaining their exponential character. As noted before, it is possible to obtain convergence to machine accuracy.

A final test consisted in computing the error between the flux integral $\int_{\Omega_{p}} J_{h} \mathrm{~d} V$ and the contour integral $\int_{\partial \Omega_{p}} \vec{h}_{h} \mathrm{~d} \vec{l}$. We show the results for the NSTX test case in Fig. 18, where it is possible to see that the two quantities are identical up to
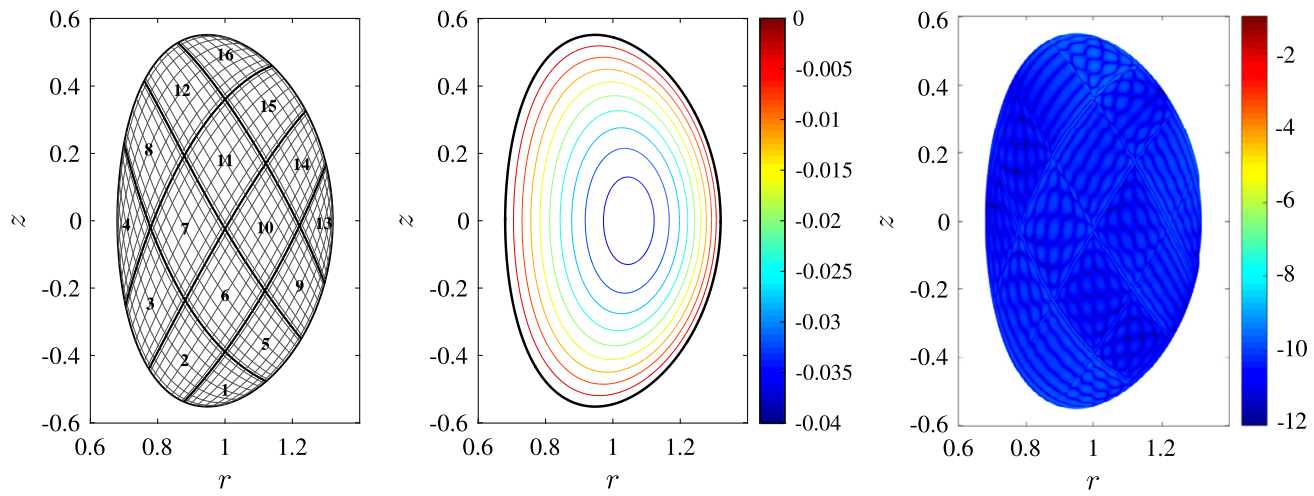

Fig. 14. Numerical solution of the Soloviev test case, (60), for ITER parameters, $\epsilon=0.32, \kappa=1.7, \delta=0.33$. From left to right: (i) computational mesh with $4 \times 4$ elements of polynomial degree $p=8$, (ii) numerical solution using the mesh in (i), $\psi_{h}$, and (iii) logarithmic error between the analytical solution and the numerical one, $\log _{10}\left|\psi_{a}-\psi_{h}\right|$.
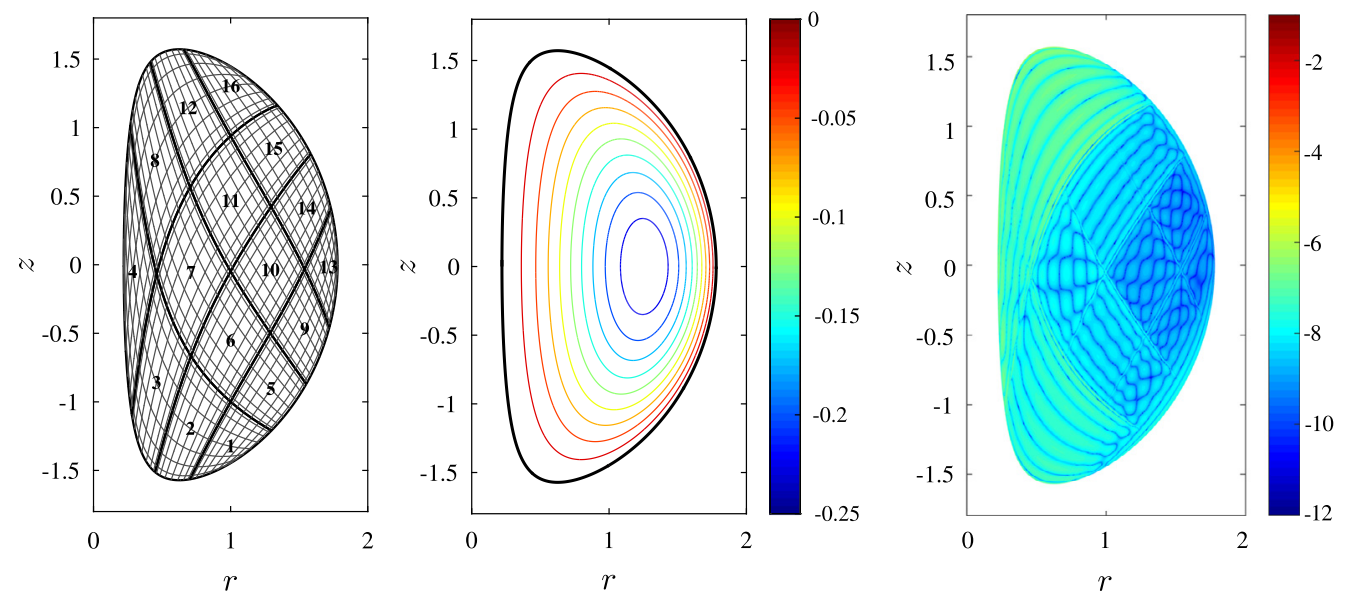

Fig. 15. Numerical solution of the Soloviev test case, (60), for NSTX parameters, $\epsilon=0.78, \kappa=2.0, \delta=0.35$. From left to right: (i) computational mesh with $4 \times 4$ elements of polynomial degree $p=8$, (ii) numerical solution using the mesh in (i), $\psi_{h}$, and (iii) logarithmic error between the analytical solution and the numerical one, $\log _{10}\left|\psi_{a}-\psi_{h}\right|$. 

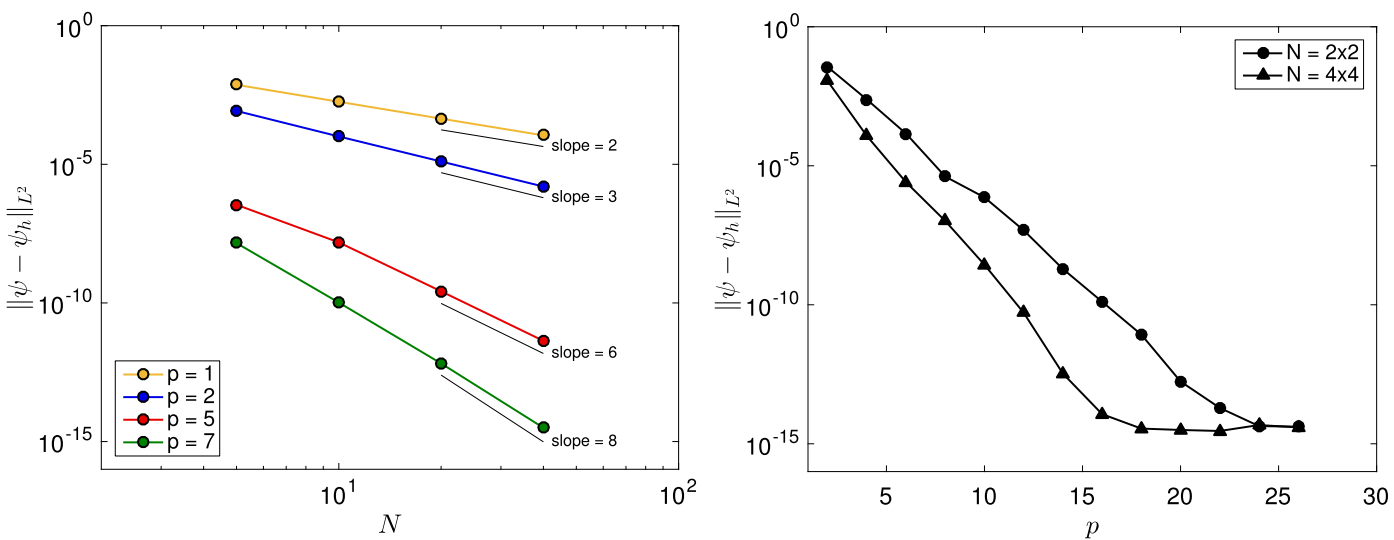

Fig. 16. Convergence plots for the numerical solution of $\psi(r, z)$ of the Soloviev test case (60) for NSTX parameters, $\epsilon=0.78, \kappa=2.0, \delta=0.35$. Left: h-convergence plots. Right: $p$-convergence plots.
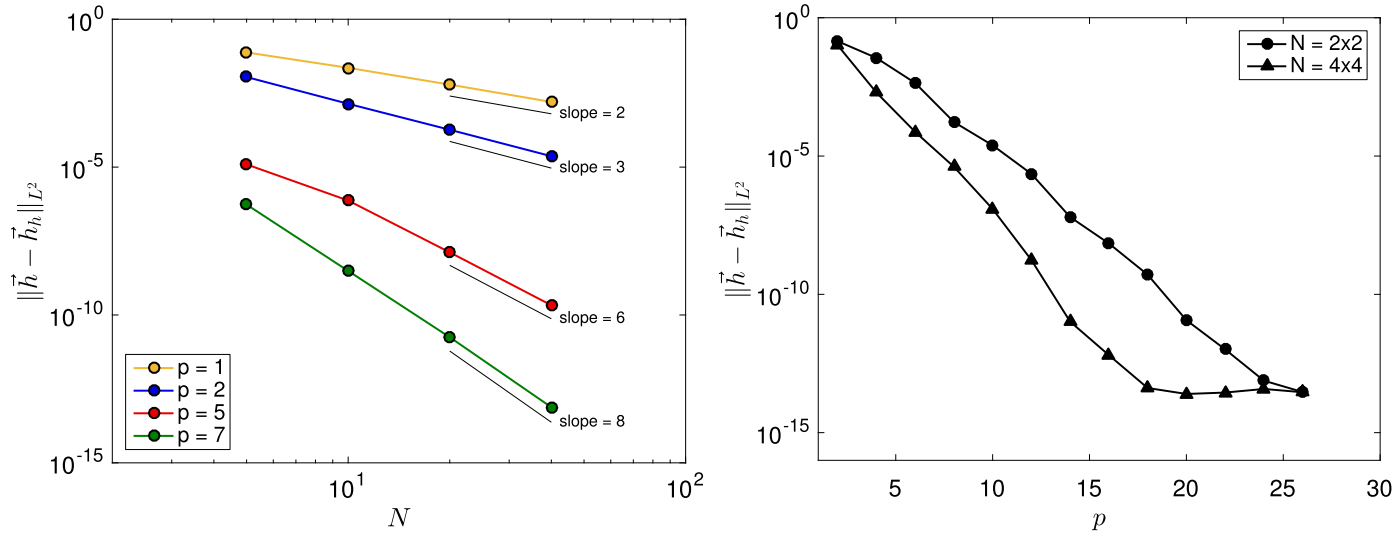

Fig. 17. Convergence plots for the numerical solution of $\vec{h}_{h}(r, z)$ of the Soloviev test case (60) for NSTX parameters, $\epsilon=0.78, \kappa=2.0, \delta=0.35$. Left: $h$-convergence plots. Right: $p$-convergence plots.
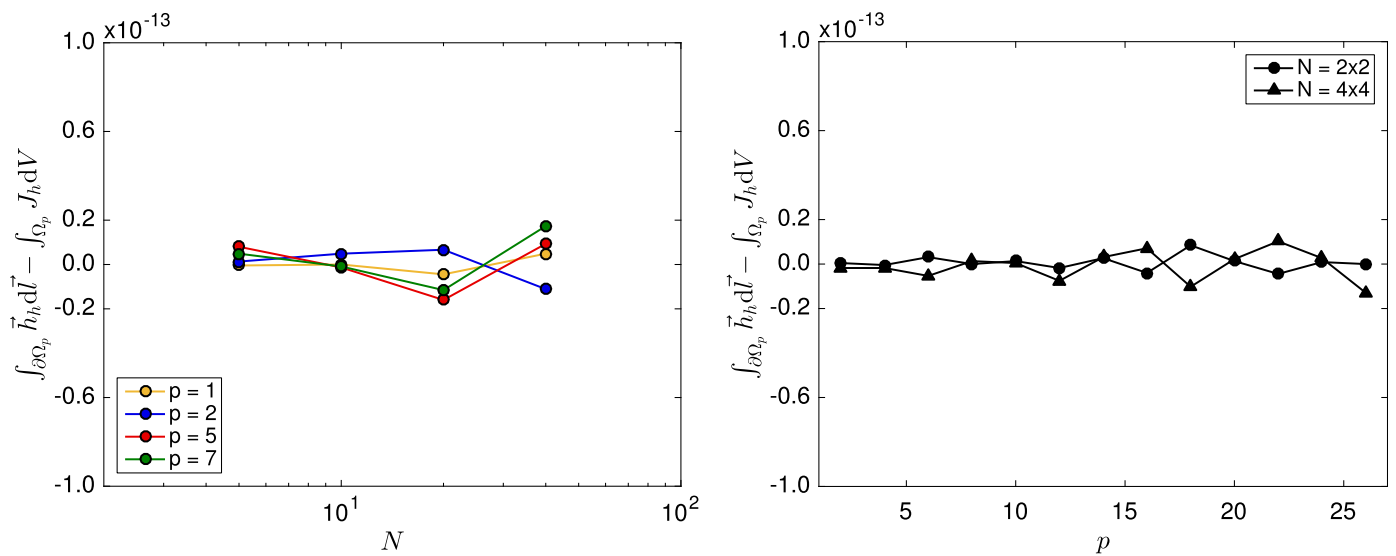

Fig. 18. Error between the flux integral $\int_{\Omega_{p}} J_{h} \mathrm{~d} V$ and the contour integral $\int_{\partial \Omega_{p}} \vec{h}_{h} \mathrm{~d} \vec{l}$, for the Soloviev test case (60) with NSTX parameters, $\epsilon=0.78, \kappa=2.0$, $\delta=0.35$. Left: $h$-convergence plots. Right: $p$-convergence plots.

machine precision. This solver can reconstruct the integral values up to machine precision, independently of the number of elements and polynomial degree of the basis functions.

\subsubsection{Linear eigenvalue problem}

For this fixed boundary test case we consider the plasma configuration used in [59], corresponding to the models $f(\psi)=0$ and $P(\psi)=\frac{C_{1}+C_{2} r^{2}}{2 \mu_{0}} \psi^{2}+P_{0}$. This leads to the following Grad-Shafranov problem 

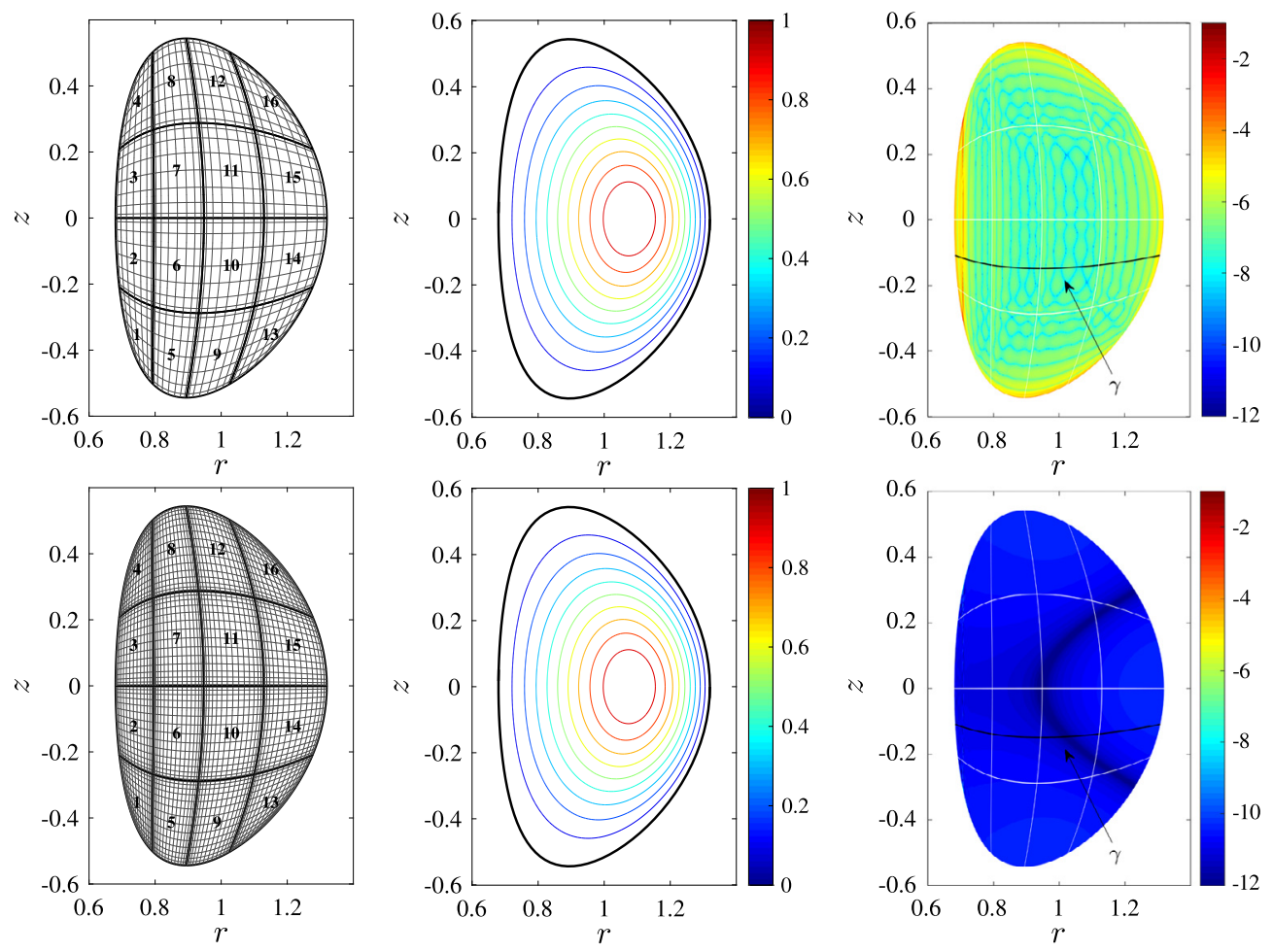

Fig. 19. Numerical solution of the linear eigenvalue ITER test case, (75), with $\left|\psi_{0}\right|=1.0, \epsilon=0.32, \kappa=1.7, \delta=0.33$ and $C_{1}=-1.0$ and $C_{2}=2.0$ (high Shafranov shift). Top: mesh of $4 \times 4$ elements and elements of polynomial degree $p=8$. Bottom: mesh of $4 \times 4$ elements and elements of polynomial degree $p=16$. From left to right: computational mesh, numerical solution, and error as given by (77).

$$
\begin{cases}\nabla \times(\mathbb{K} \nabla \times \psi)=\left(\frac{C_{1}}{r}+C_{2} r\right) \frac{\psi}{\mu_{0}} & \text { in } \Omega_{p}, \\ \psi=0 & \text { on } \partial \Omega_{p},\end{cases}
$$

with the plasma boundary $\Omega_{p}$ given by

$$
\left\{\begin{array}{lll}
r(s)=2+\epsilon \cos (s+\alpha \sin s) & \text { with } & s \in[0,2 \pi[, \\
z(s)=\epsilon \kappa \sin s & \text { with } & s \in[0,2 \pi[.
\end{array}\right.
$$

The coefficients $C_{i}$ determine the Shafranov shift of the solutions. Several different values were tested but here we only present the results corresponding to $C_{1}=-1.0$ and $C_{2}=2.0$, the highest Shafranov shift used in [59]. Two plasma shapes have been tested: (i) $\epsilon=0.32, \kappa=1.7$ and $\delta=0.33$ (ITER shape, Fig. 19), and (ii) $\epsilon=0.78, \kappa=2.0$ and $\delta=0.45$ (NSTX shape, Fig. 20).

Since analytical solutions are not available for these test cases, it is not possible to compute the exact error associated to the numerical approximation. In order to assess the accuracy of the method in these cases, we compute and analyze the following error term

$$
\mathrm{E}=\left|\frac{\nabla \times \vec{h}_{h}-\sigma J\left(r, z, \psi_{h}\right)}{\sigma J\left(r, z, \psi_{h}\right)}\right| .
$$

We can see, Fig. 19 and Fig. 20, that the method presented in this article can accurately reproduce the plasma contour lines on both meshes, even for highly elongated plasmas. The error is substantially reduced when the polynomial degree of the basis functions is increased, as has already been seen in the previous test cases.

For the ease of comparison, the error (77) along the line $\gamma$ (see Fig. 19 for ITER test case and Fig. 20 for NSTX test case) is shown in Fig. 21.

\subsubsection{Non-linear eigenvalue problem}

To further assess the proposed numerical model on a fully non-linear problem, we apply it to the non-linear test case presented in [59]. This test case corresponds to a configuration with a pressure pedestal and consists of the following models: 

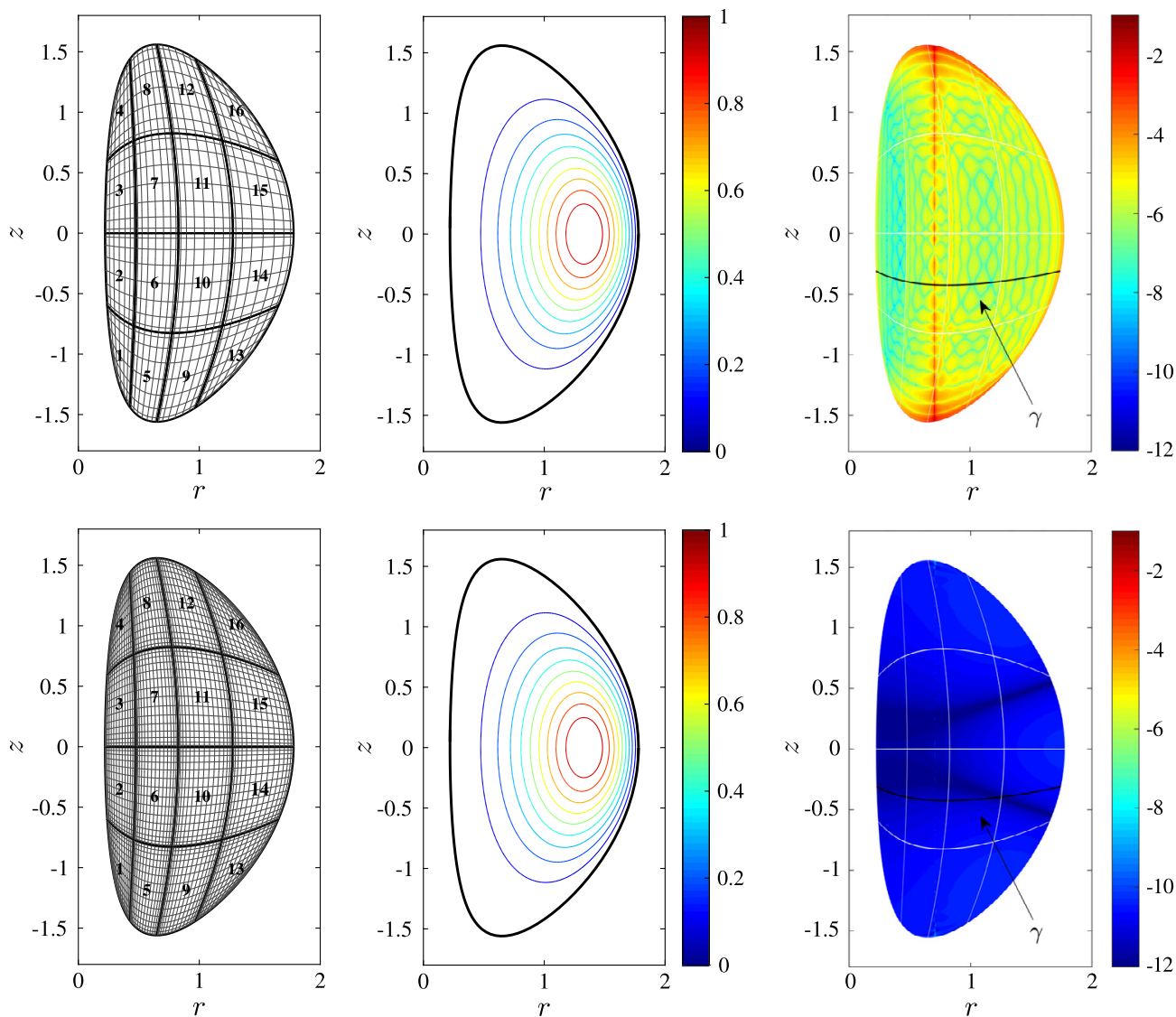

Fig. 20. Numerical solution of the linear eigenvalue NSTX test case, (75), with $\left|\psi_{0}\right|=1.0, \epsilon=0.78, \kappa=2.0, \delta=0.45$ and $C_{1}=-1.0$ and $C_{2}=2.0$ (high Shafranov shift). Top: mesh of $4 \times 4$ elements and elements of polynomial degree $p=8$. Bottom: mesh of $4 \times 4$ elements and elements of polynomial degree $p=16$. From left to right: computational mesh, numerical solution, and error as given by (77).
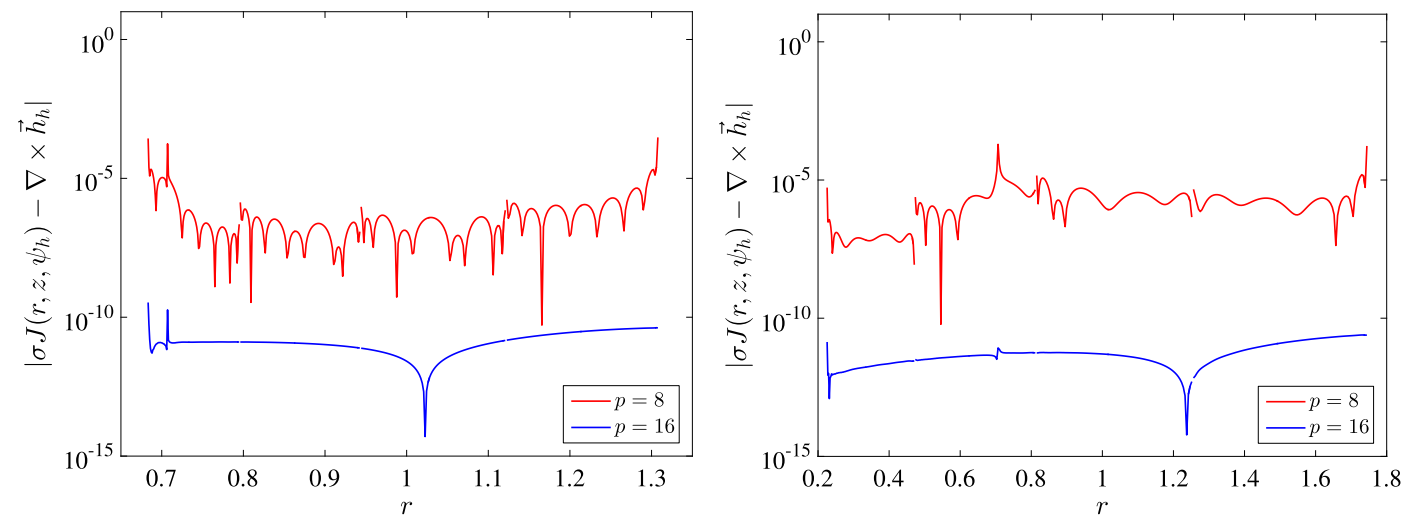

Fig. 21. Error, as given by (77), along the curve $\gamma$ (see Fig. 19 for ITER test case and Fig. 20 for NSTX test case). Left: ITER test case. Right: NSTX test case.

$$
f(\psi)=0 \quad \text { and } \quad P(\psi)=\frac{C_{1}+C_{2} \psi^{2}}{\mu_{0}}\left(1-e^{-\frac{\psi^{2}}{\eta}}\right),
$$

which lead to the following non-linear Grad-Shafranov problem

$$
\begin{cases}\nabla \times(\mathbb{K} \nabla \times \psi)=2 r\left[C_{2} \psi\left(1-e^{-\frac{\psi^{2}}{\eta}}\right)+\frac{C_{1}+C_{2} \psi^{2}}{\eta} \psi \eta^{-\frac{\psi^{2}}{\eta}}\right] & \text { in } \Omega_{p}, \\ \psi=0 & \text { on } \partial \Omega_{p},\end{cases}
$$

with the plasma boundary $\Omega_{p}$ given by (76). 

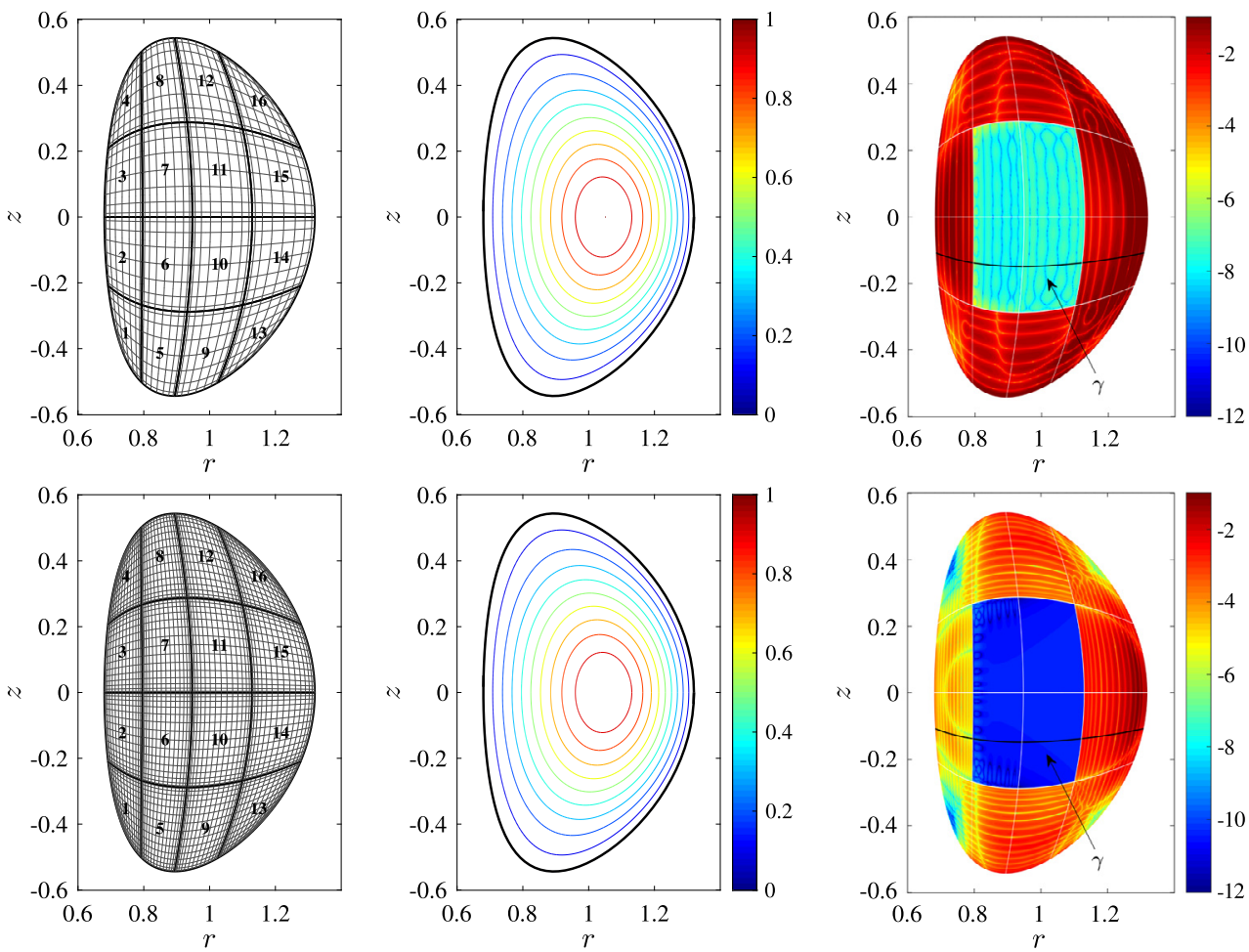

Fig. 22. Numerical solution of the non-linear eigenvalue ITER test case, (79), with $\left|\psi_{0}\right|=1.0, \epsilon=0.32, \kappa=1.7, \delta=0.33$ and $C_{1}=0.8$ and $C_{2}=0.2$ and $\eta=0.1$. Top: mesh of $4 \times 4$ elements and elements of polynomial degree $p=8$. Bottom: mesh of $4 \times 4$ elements and elements of polynomial degree $p=16$. From left to right: computational mesh, numerical solution, and error as given by (77).

For the coefficients $C_{i}$ we use the same values as in [59], $C_{1}=0.8$ and $C_{2}=0.2$, and we set $\eta=0.1$. ITER (Fig. 22) and NSTX (Fig. 23) plasma shapes, as in Section 3.2.2, are tested.

As can be seen in Fig. 22 and Fig. 23, an increase in the polynomial degree of the basis functions leads to a reduction of the error in the numerical solution.

An interesting aspect of this test case is that for these meshes the elements at the boundary show a substantially larger error than the interior elements. This behavior can be explained by analyzing $\nabla \times \vec{h}_{h}(r, z)$ and $\sigma J\left(r, z, \psi_{h}\right)$. In Fig. 24 we compare $\sigma J\left(r, z, \psi_{h}\right)$ to $\nabla \times \vec{h}_{h}(r, z)$ for $p=8,16$ along the line $\gamma$ (Fig. 22 for ITER case and Fig. 23 for NSTX case). As can be seen, for the ITER shape there is a sharp variation of $\sigma J\left(r, z, \psi_{h}\right)$ close to the edge of the plasma. This sharp variation cannot be accurately recovered with $p=8$ for large elements, as used here. Nevertheless, with an increase of the polynomial degree to $p=16$, we obtain a much better agreement, see Fig. 25 .

\subsection{Plasma shape with an $X$-point}

The final test cases correspond to a plasma with an X-point, leading to a plasma boundary with a sharp corner. This plasma configuration will be assessed for a Soloviev problem, the linear eigenvalue problem introduced in Section 3.2.2 and the non-linear eigenvalue problem of Section 3.2.3.

\subsubsection{Plasma shape definition}

The shape of the plasma considered here is the up-down asymmetric ITER-like configuration presented in [15], see Fig. 26. This shape is given by the equation

$$
\frac{r^{4}}{8}+A\left(\frac{r^{2}}{2} \ln r-\frac{r^{4}}{8}\right)+\sum_{k=1}^{12} c_{k} \psi_{k}(r, z)=0,
$$

with the functions $\psi_{i}(r, z)$ and the coefficients $c_{i}$ as in Appendix A.

\subsubsection{Soloviev problem}

The first test case for the X-point plasma corresponds to a Soloviev solution of the Grad-Shafranov equation. This special case corresponds to $f(\psi)=\sqrt{2 \mu_{0} A \psi}$ combined with a linear model $P(\psi)=(1-A) \psi$, for a more detailed discussion see [15]. This leads to the following Grad-Shafranov equation 

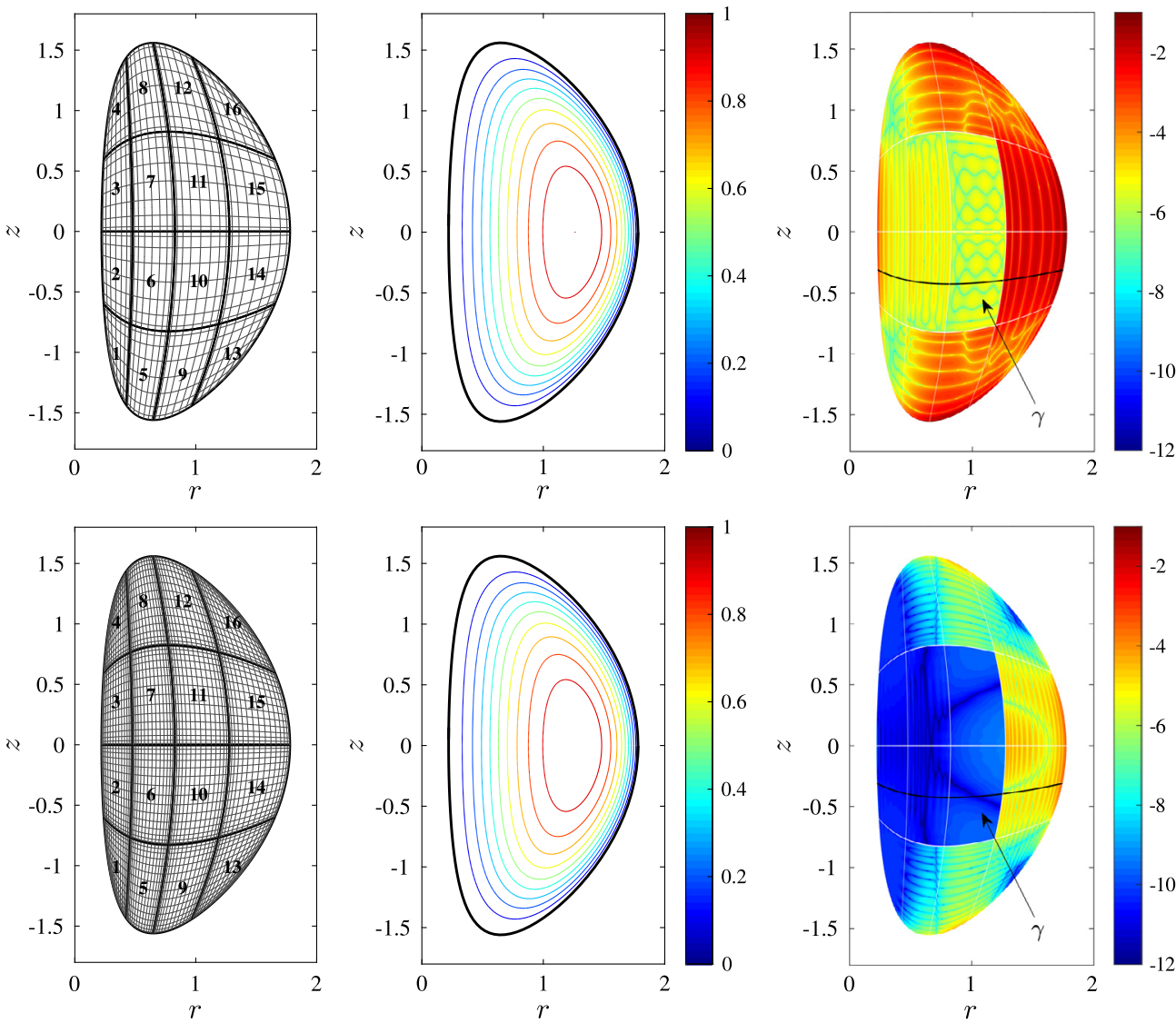

Fig. 23. Numerical solution of the non-linear eigenvalue NSTX test case, (79), with $\left|\psi_{0}\right|=1.0, \epsilon=0.78, \kappa=2.0, \delta=0.45, C_{1}=0.8, C_{2}=0.2$ and $\eta=0.1$. Top: mesh of $4 \times 4$ elements and elements of polynomial degree $p=8$. Bottom: mesh of $4 \times 4$ elements and elements of polynomial degree $p=16$. From left to right: computational mesh, numerical solution, and error as given by (77).
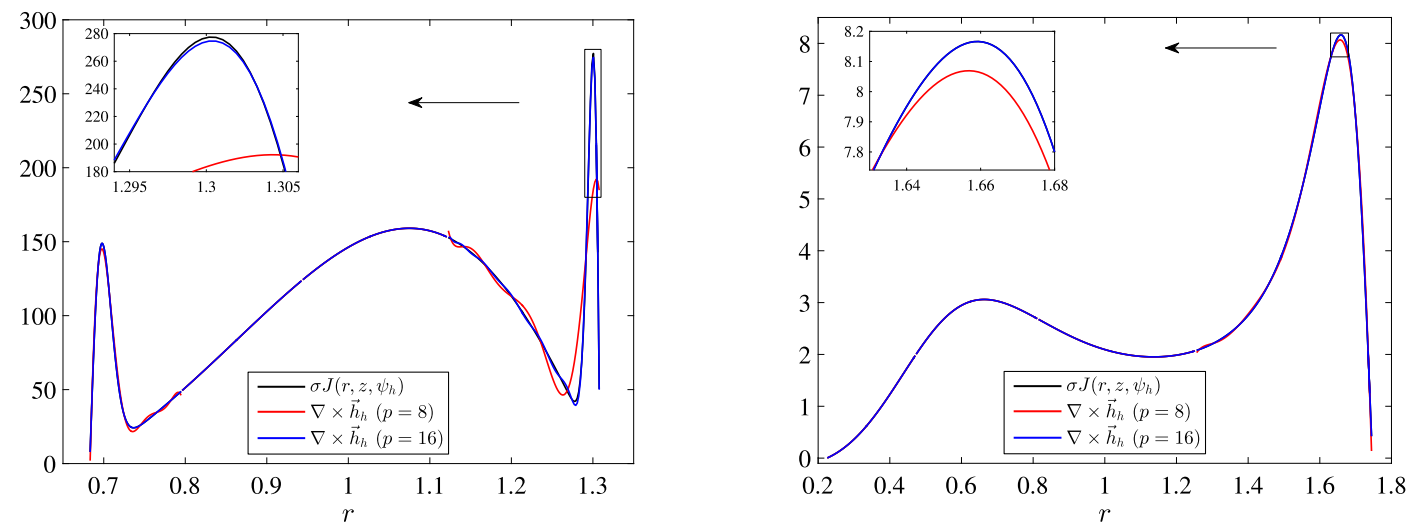

Fig. 24. Comparison between $\sigma J\left(r, z, \psi_{h}\right)$ and $\nabla \times \vec{h}_{h}(r, z)$ for $p=8,16$ along the line $\gamma$ (Fig. 22 for ITER case and Fig. 23 for NSTX case). Left: ITER test case. Right: NSTX test case.

$$
\begin{cases}\nabla \times(\mathbb{K} \nabla \times \psi)=(1-A) r+\frac{A}{r} & \text { in } \quad \Omega_{p}, \\ \psi=0 & \text { on } \partial \Omega_{p},\end{cases}
$$

where we will consider $A=-0.155$.

The analytical solution, $\psi_{a}(r, z)$, is constructed by adding to the particular solution $\frac{r^{4}}{8}+A\left(\frac{r^{2}}{2} \ln r-\frac{r^{4}}{8}\right)$ a linear combination of twelve homogeneous solutions, $\psi_{i}(r, z)$ with $i=1, \ldots, 12$ : 

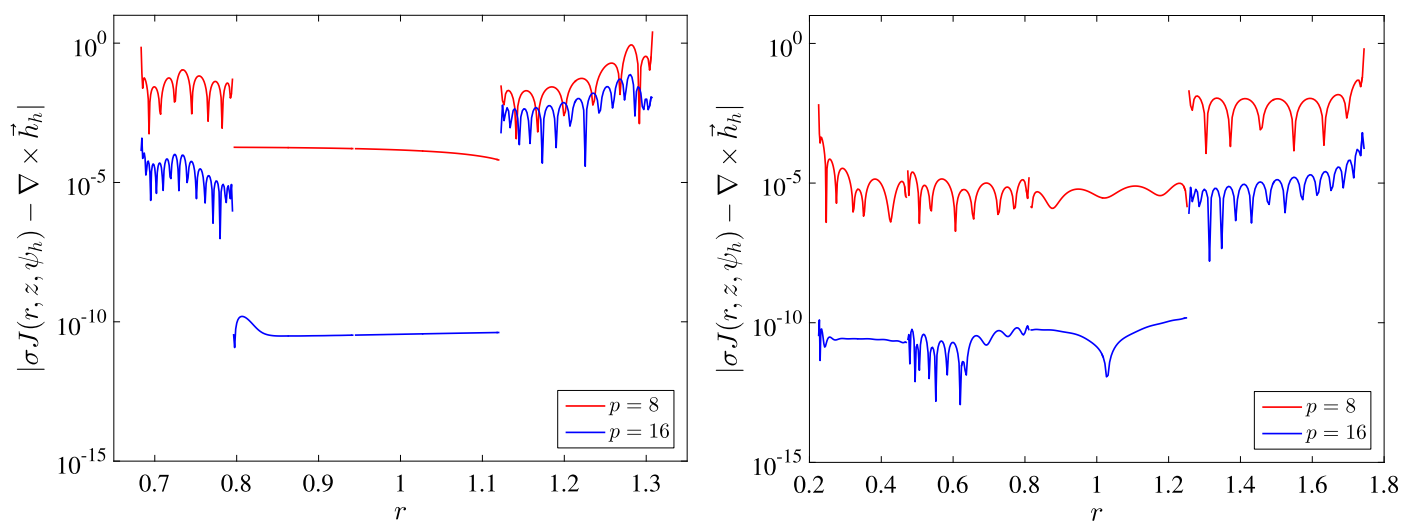

Fig. 25. Error, as given by (77), along the curve $\gamma$ (see Fig. 22 for ITER test case and Fig. 23 for NSTX test case). Left: ITER test case. Right: NSTX test case.

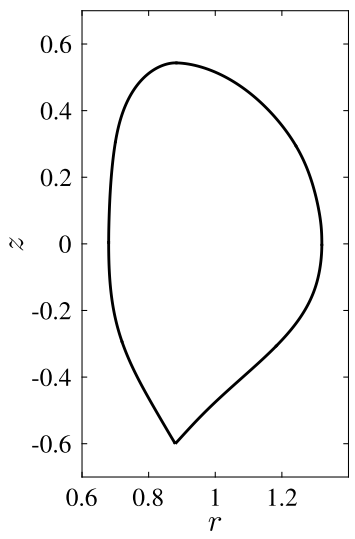

Fig. 26. Plasma shape with an X-point, corresponding to the up-down asymmetric ITER-like configuration presented in [15].
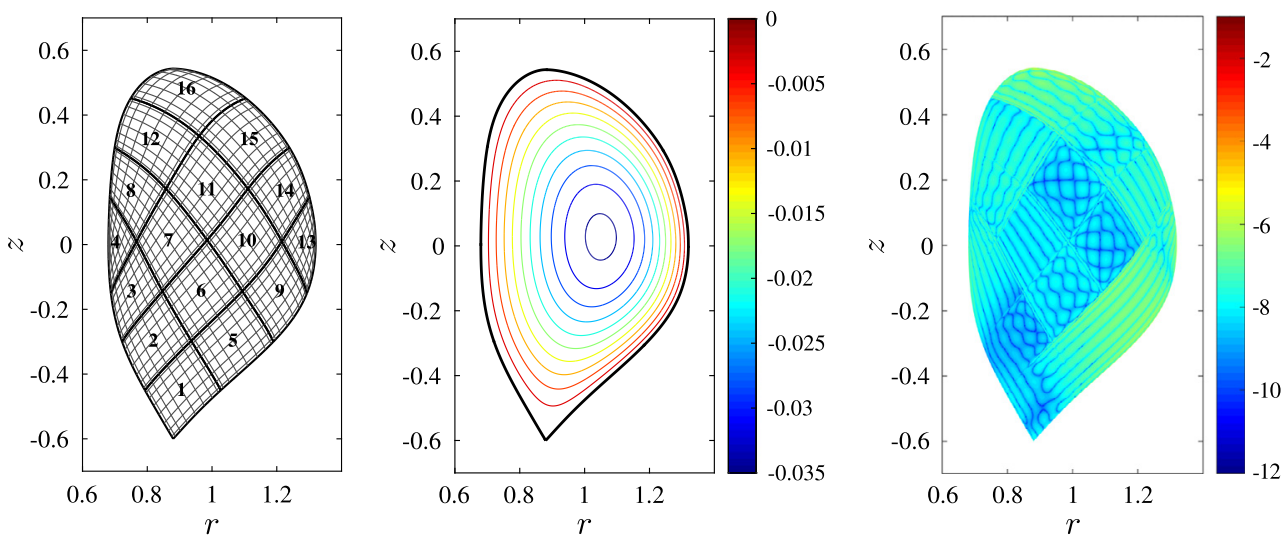

Fig. 27. Numerical solution of the X-point Soloviev test case, (81). From left to right: (i) computational mesh with $4 \times 4$ elements of polynomial degree $p=8$, (ii) numerical solution using the mesh in (i), $\psi_{h}$, and (iii) logarithmic error between the analytical solution and the numerical one, $\log _{10}\left|\psi_{a}-\psi_{h}\right|$.

$$
\psi_{a}(r, z)=\frac{r^{4}}{8}+A\left(\frac{r^{2}}{2} \ln r-\frac{r^{4}}{8}\right)+\sum_{k=1}^{12} c_{k} \psi_{k}(r, z),
$$

with the homogeneous solutions and the coefficients $c_{i}$ as given in Appendix A. The methodology to obtain this solution is similar to the one presented in Section 3.1.1 and is fully detailed in [15].

In Fig. 27 an example solution with $4 \times 4$ elements of polynomial degree $p=8$ is presented. As can be seen, the solution is well reconstructed by the proposed method.

The convergence tests for $h$-refinement and $p$-refinement show very similar results to the ITER and NSTX cases of Section 3.1.1, both for $\psi_{h}$ and for $\vec{h}_{h}$, see Fig. 28 and Fig. 29. As seen before, accuracy up to machine precision is achieved. 

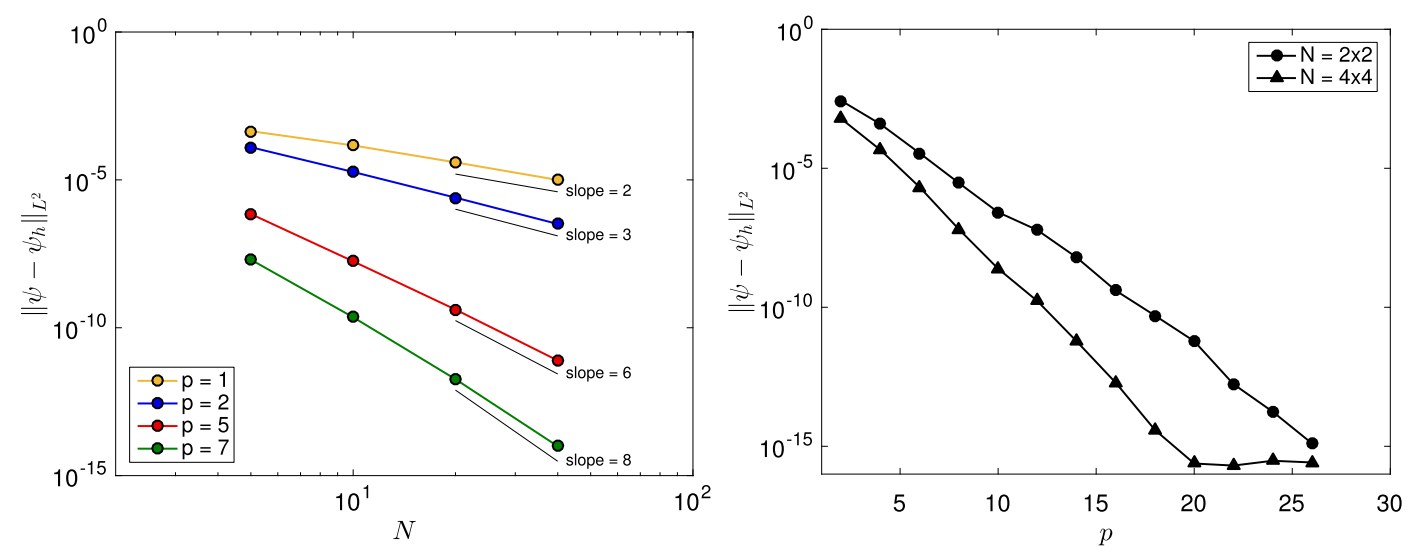

Fig. 28. Convergence plots for the numerical solution of $\psi(r, z)$ of the X-point Soloviev test case (81). Right: $p$-convergence plots.
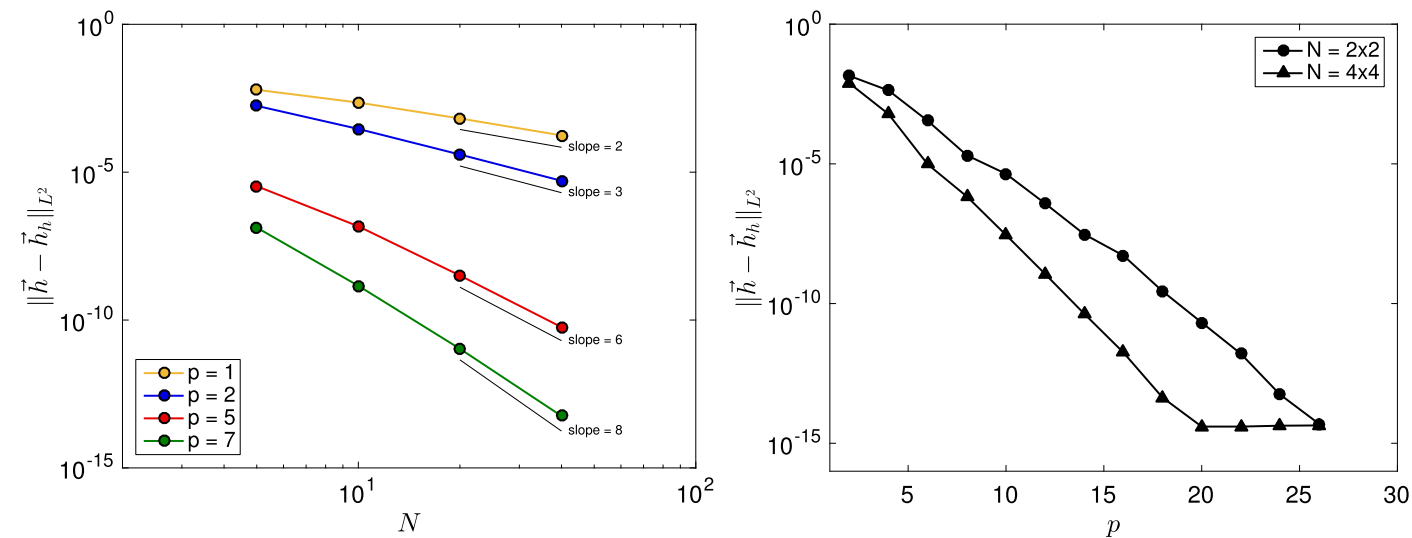

Fig. 29. Convergence plots for the numerical solution of $\vec{h}_{h}(r, z)$ of the X-point Soloviev test case (81). Left: $h$-convergence plots. Right: $p$-convergence plots.
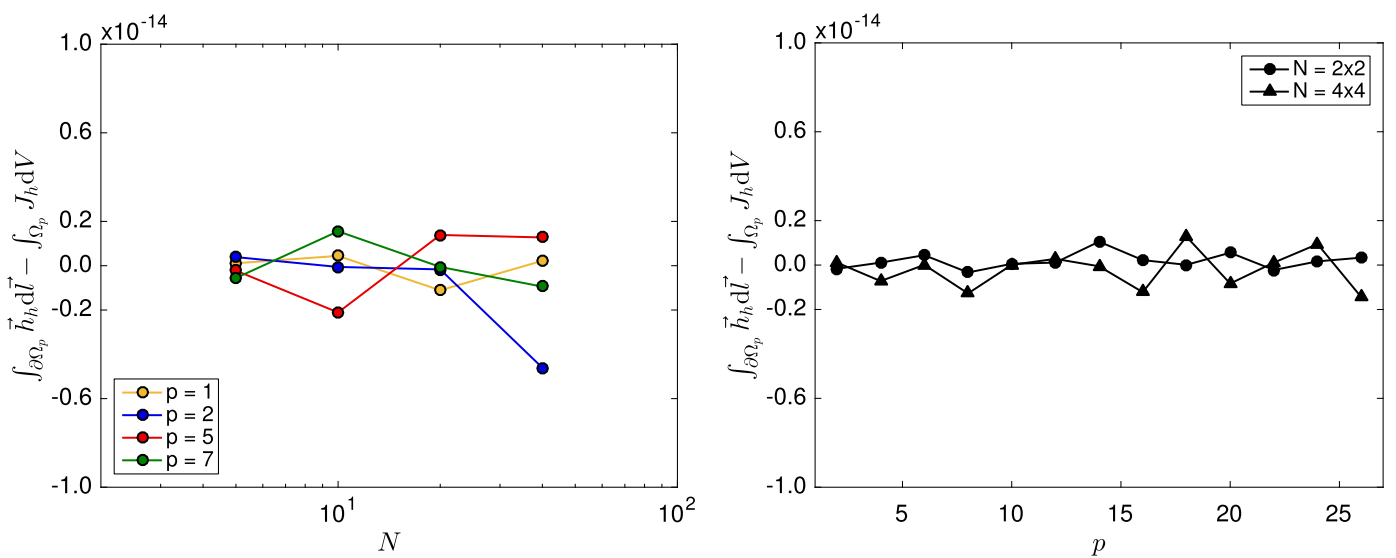

Fig. 30. Error between the flux integral $\int_{\Omega_{p}} J_{h} \mathrm{~d} V$ and the contour integral $\int_{\partial \Omega_{p}} \vec{h}_{h} \mathrm{~d} \vec{l}$, for the X-point Soloviev test case (81). Left: $h$-convergence plots. Right: $p$-convergence plots.

As in Section 3.1.1, the error between the flux integral $\int_{\Omega_{p}} J_{h} \mathrm{~d} V$ and the contour integral $\int_{\partial \Omega_{p}} \vec{h}_{h} \mathrm{~d} \vec{l}$ shows excellent results, see Fig. 30.

\subsubsection{Linear eigenvalue problem}

The second test for the X-point plasma configuration is the linear eigenvalue problem of Section 3.2.2. The same GradShafranov problem in (75) with $C_{1}=-1.0$ and $C_{2}=2.0$ is solved with the plasma boundary $\Omega_{p}$ given by (80). 

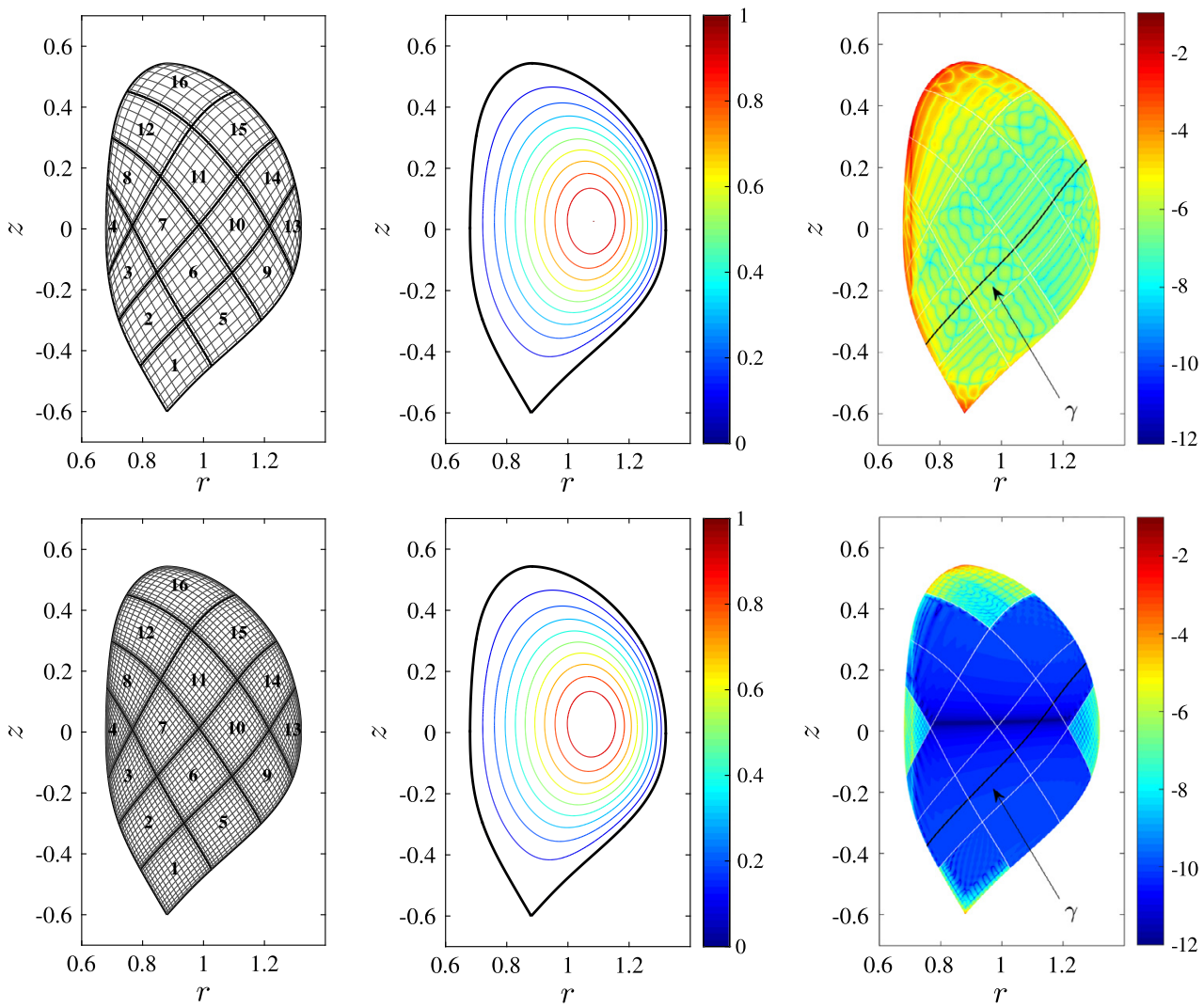

Fig. 31. Numerical solution of the linear eigenvalue problem (75) for the X-point plasma shape given in (80), with $\left|\psi_{0}\right|=1.0, C_{1}=-1.0$ and $C_{2}=2.0$ (high Shafranov shift). Top: mesh of $4 \times 4$ elements and elements of polynomial degree $p=8$. Bottom: mesh of $4 \times 4$ elements and elements of polynomial degree $p=16$. From left to right: computational mesh, numerical solution, and error as given by (77).

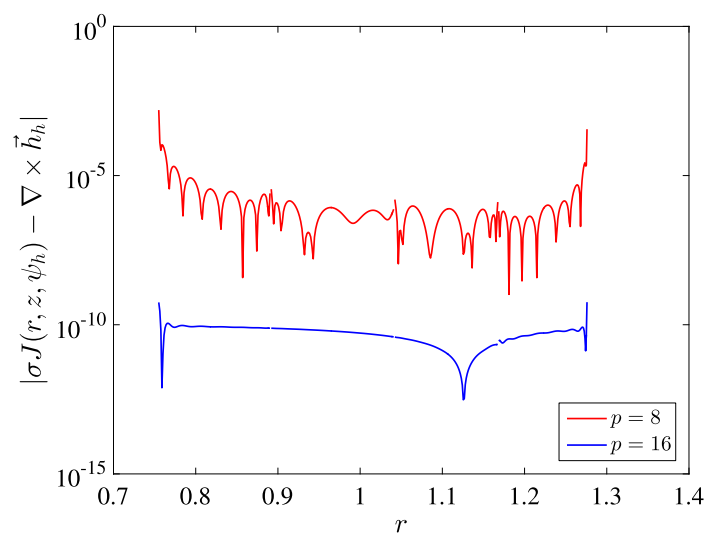

Fig. 32. Error, as given by (77), along the curve $\gamma$ (see Fig. 31).

We first show in Fig. 31 two example solutions obtained with a mesh of $4 \times 4$ elements of polynomial degree $p=8$ and $p=16$. As can be seen the error $E$ given by (77) considerably decreases when the polynomial degree of the elements is increased from $p=8$ to $p=16$.

For the ease of comparison, the error (77) along the line $\gamma$ (see Fig. 31) is shown in Fig. 32. It is possible to see that the proposed method can accurately solve the linear eigenvalue Grad-Shafranov problem for a plasma with an X-point.

\subsubsection{Non-linear eigenvalue problem}

The third and final test case for the X-point plasma configuration is the non-linear Grad-Shafranov problem of Section 3.2.3 with $\eta=0.1$. The difference between this case and the previously discussed cases is the X-point plasma shape. 

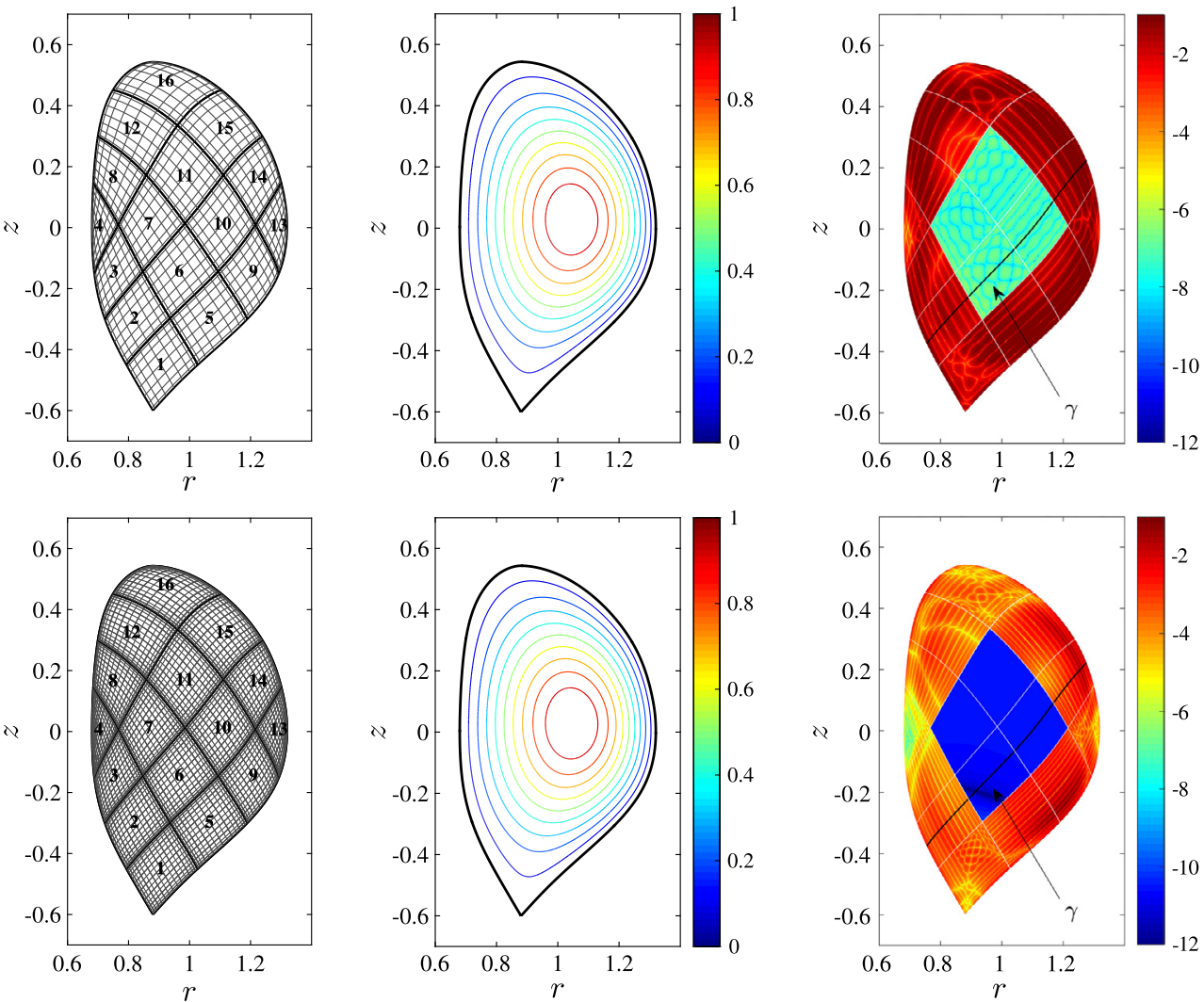

Fig. 33. Numerical solution of the non-linear eigenvalue X-point test case, (79), with $\left|\psi_{0}\right|=1.0$ and $\eta=0.1$. Top: mesh of $4 \times 4$ elements and elements of polynomial degree $p=8$. Bottom: mesh of $4 \times 4$ elements and elements of polynomial degree $p=16$. From left to right: computational mesh, numerical solution, and error as given by (77).
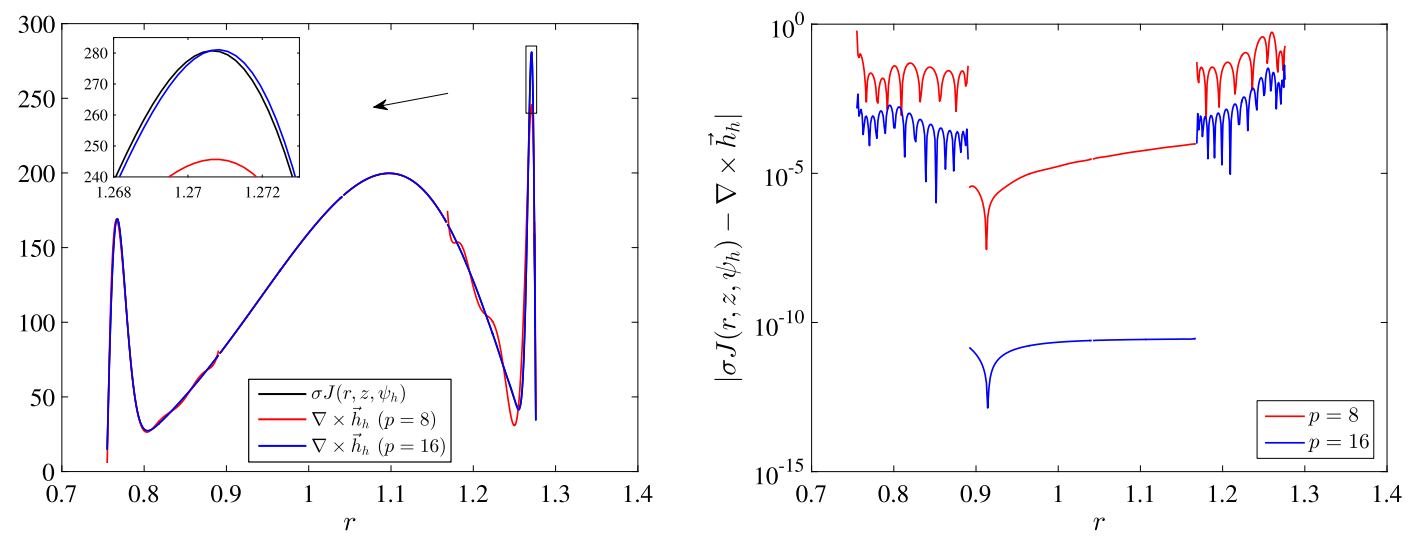

Fig. 34. Left: Comparison between $\sigma J\left(r, z, \psi_{h}\right)$ and $\nabla \times \vec{h}_{h}$ for $p=8,16$, along the curve $\gamma$ (see Fig. 33). Right: Error, as given by (77), along the curve $\gamma$ (see Fig. 33).

Numerical solutions corresponding to a mesh with $4 \times 4$ elements of polynomial degree $p=8$ and $p=16$ are shown in Fig. 33. As can be seen, the mimetic spectral element solver can adequately compute the non-linear equilibrium solution for a plasma with an X-point, showing a similar error to the cases discussed in Section 3.2.3.

As seen in Section 3.2.3, for these meshes the elements at the boundary show a substantially larger error than the interior ones. This is again due to the large gradient in $\sigma J\left(r, z, \psi_{h}\right)$. In Fig. 34 we compare $\sigma J\left(r, z, \psi_{h}\right)$ to $\nabla \times \vec{h}_{h}(r, z)$ for $p=8,16$ along the line $\gamma$, see Fig. 33. As can be seen, there is a sharp variation of $\sigma J\left(r, z\right.$, $\left.\psi_{h}\right)$ close to the edge of the plasma. The error (77) along the line $\gamma$ (see Fig. 33) is shown in Fig. 34 right. It is possible to see a substantial reduction in the error when the polynomial degree of the elements is increased from $p=8$ to $p=16$. 


\section{Conclusions}

This article presents a new, fixed-boundary, Eulerian Grad-Shafranov solver based on the mimetic spectral element framework $[44,56]$. The advantages of this method are: (i) high order accuracy, enabling both spectral and geometric convergence, (ii) geometric flexibility, allowing for arbitrary plasma shapes (symmetric, asymmetric and including X-points) and (iii) exact discretization of topological laws, resulting in an exact computation of the total plasma current.

We have shown for a wide range of profiles, including fusion relevant profiles with a pressure pedestal and high Shafranov shift, that the proposed method is capable of accurately computing equilibria. Highly accurate solutions of the order $10^{-15}$ (machine precision) can be achieved with relatively coarse meshes, using high order basis functions. This characteristic represents one of the attractions of spectral element based discretizations: small numerical errors can be obtained with a much smaller number of degrees of freedom using higher order basis functions.

Nevertheless, the proposed method still allows for further improvement, mainly in terms of speed. An important improvement to this method would be the use of a Newton solver instead of the Picard iteration presented. Another possible improvement is the optimization of the discretization of the right-hand side, $J_{\phi}(r, z, \psi)$, required in each iteration. These improvements are the subject of ongoing and future research.

\section{Appendix A. X-point Soloviev solution}

The X-point Soloviev solution used in this work corresponds to the up-down asymmetric ITER-like configuration presented in [15]. The analytical solution is given by

$$
\psi_{a}(r, z)=\frac{r^{4}}{8}+A\left(\frac{r^{2}}{2} \ln r-\frac{r^{4}}{8}\right)+\sum_{k=1}^{12} c_{k} \psi_{k}(r, z),
$$

with

$$
\begin{aligned}
& \psi_{1}(r, z)=1 \\
& \psi_{2}(r, z)=r^{2} \\
& \psi_{3}(r, z)=z^{2}-r^{2} \ln r \\
& \psi_{4}(r, z)=r^{4}-4 r^{2} z^{2} \\
& \psi_{5}(r, z)=2 z^{4}-9 z^{2} r^{2}+3 r^{4} \ln r-12 r^{2} z^{2} \ln r \\
& \psi_{6}(r, z)=r^{6}-12 r^{4} z^{2}+8 r^{2} z^{4} \\
& \psi_{7}(r, z)=8 z^{6}-140 z^{4} r^{2}+75 z^{2} r^{4}-15 r^{6} \ln r+180 r^{4} z^{2} \ln r-120 r^{2} z^{4} \ln r, \\
& \psi_{8}(r, z)=z \\
& \psi_{9}(r, z)=z r^{2} \\
& \psi_{10}(r, z)=z^{3}-3 z r^{2} \ln r \\
& \psi_{11}(r, z)=3 z r^{4}-4 z^{3} r^{2}, \\
& \psi_{12}(r, z)=8 z^{5}-45 z r^{4}-80 z^{3} r^{2} \ln r+60 z r^{4} \ln r,
\end{aligned}
$$

and the values for the $c_{i}$ coefficients given in Table A.2.

Table A.2

Values for the $c_{i}$ coefficients for the up-down asymmetric ITER-like configuration of (A.1).

\begin{tabular}{rr|rr|rr}
\hline$c_{1}:$ & 0.0864912785478807 & $c_{5}:$ & 0.3807375276922255 & $c_{9}:$ & 0.7401867427139835 \\
$c_{2}:$ & 0.3236475999311713 & $c_{6}:$ & -0.3573346678775972 & $c_{10}:$ & -0.4397718916520960 \\
$c_{3}:$ & -0.5227047152014734 & $c_{7}:$ & -0.0148740157319066 & $c_{11}:$ & -0.1071308624644806 \\
$c_{4}:$ & -0.2319735789049367 & $c_{8}:$ & 0.1480149379993163 & $c_{12}:$ & 0.0127862151469652 \\
\hline
\end{tabular}

\section{References}

[1] R. Abraham, J.E. Marsden, T. Ratiu, Manifolds, Tensor Analysis, and Applications, Applied Mathematical Sciences, vol. 75, Springer, 2001.

[2] R. Albanese, R. Ambrosino, M. Mattei, CREATE-NL+: a robust control-oriented free boundary dynamic plasma equilibrium solver, Fusion Eng. Des. 96-97 (2015) 664-667.

[3] J.F. Artaud, V. Basiuk, F. Imbeaux, M. Schneider, J. Garcia, G. Giruzzi, P. Huynh, T. Aniel, F. Albajar, J.M. Ané, A. Bécoulet, C. Bourdelle, A. Casati, L. Colas, J. Decker, R. Dumont, L.G. Eriksson, X. Garbet, R. Guirlet, P. Hertout, G.T. Hoang, W. Houlberg, G. Huysmans, E. Joffrin, S.H. Kim, F. Köchl, J. Lister, X. Litaudon, P. Maget, R. Masset, B. Pégourié, Y. Peysson, P. Thomas, E. Tsitrone, F. Turco, The CRONOS suite of codes for integrated tokamak modelling, Nucl. Fusion 50 (2010) 043001. 
[4] P.M. Bellan, Generalization of cylindrical spheromak solution to finite beta and large reversed shear, Phys. Plasmas 9 (2002) 3050.

[5] J. Blum, J. Le Foll, Plasma equilibrium evolution at the resistive diffusion timescale, Comput. Phys. Rep. 1 (1984) $465-494$.

[6] A. Bossavit, Computational electromagnetism and geometry: (1) Network equations, J. Jpn. Soc. Appl. Electromagn. 7 (1999) $150-159$.

[7] A. Bossavit, Computational electromagnetism and geometry: (2) Network constitutive laws, J. Jpn. Soc. Appl. Electromagn. 7 (1999) $294-301$.

[8] A. Bossavit, Computational electromagnetism and geometry: (4) From degrees of freedom to fields, J. Jpn. Soc. Appl. Electromagn. 8 (2000) 102-109.

[9] A. Bossavit, Computational electromagnetism and geometry: (5) The "Galerkin hodge", J. Jpn. Soc. Appl. Electromagn. 8 (2000) $203-209$.

[10] M. Bouman, A. Palha, J. Kreeft, M. Gerritsma, A conservative spectral element method for curvilinear domains, in: Spectral and High Order Methods for Partial Differential Equations, in: Lecture Notes in Computational Science and Engineering, vol. 76, Springer, 2011, pp. 111-119.

[11] M. Brambilla, Numerical simulation of ion cyclotron waves in tokamak plasmas, Plasma Phys. Control. Fusion 41 (1999) 1-34.

[12] F. Brezzi, M. Fortin, Mixed and Hybrid Finite Element Methods, Springer Series in Computational Mathematics, vol. 15, Springer, 1991.

[13] R.V. Budny, M.G. Bell, H. Biglari, M. Bitter, C.E. Bush, C.Z. Cheng, E.D. Fredrickson, B. Grek, K.W. Hill, H. Hsuan, A.C. Janos, D.L. Jassby, D.W. Johnson, L.C. Johnson, B. LeBlanc, D.C. McCune, D.R. Mikkelsen, H.K. Park, A.T. Ramsey, S.A. Sabbagh, S.D. Scott, J.F. Schivell, J.D. Strachan, B.C. Stratton, E.J Synakowski, G. Taylor, M.C. Zarnstorff, S.J. Zweben, Simulations of deuterium-tritium experiments in TFTR, Nucl. Fusion 32 (1992) $429-447$.

[14] G. Cenacchi, A. Taroni, JETTO a free boundary plasma transport code, Tech. rep., ENEA, 1988.

[15] A.J. Cerfon, J.P. Freidberg, “One size fits all” analytic solutions to the Grad-Shafranov equation, Phys. Plasmas 17 (2010) 032502.

[16] D.P. Coster, V. Basiuk, G. Pereverzev, D. Kalupin, R. Zagorksi, R. Stankiewicz, P. Huynh, F. Imbeaux, The European Transport Solver, IEEE Trans. Plasma Sci. 38 (2010) 2085-2092.

[17] J.A. Crotinger, L. LoDestro, L.D. Pearlstein, A. Tarditi, T.A. Casper, E.B. Hooper, Corsica: a comprehensive simulation of toroidal magnetic-fusion devices, Tech. rep., Final Report to the LDRD program, 1997.

[18] O. Czarny, G. Huysmans, Bézier surfaces and finite elements for MHD simulations, J. Comput. Phys. 227 (2008) $7423-7445$.

[19] E. Fable, C. Angioni, A. Ivanov, K. Lackner, O. Maj, S. Yu, G. Pautasso, G. Pereverzev, A stable scheme for computation of coupled transport and equilibrium equations in tokamaks, Nucl. Fusion 53 (2013) 033002.

[20] F. Felici, O. Sauter, S. Coda, B.P. Duval, T.P. Goodman, J.-M. Moret, J.I. Paley, Real-time physics-model-based simulation of the current density profile in tokamak plasmas, Nucl. Fusion 51 (2011) 083052.

[21] T. Frankel, The Geometry of Physics, Cambridge University Press, 2004

[22] M. Gerritsma, Edge functions for spectral element methods, in: Spectral and High Order Methods for Partial Differential Equations, in: Lecture Notes in Computational Science and Engineering, vol. 76, Springer, 2011, pp. 199-207.

[23] M. Gerritsma, M. Bouman, A. Palha, Least-squares spectral element method on a staggered grid, in: Large-Scale Scientific Computing, in: Lecture Notes in Computer Science, vol. 5910, Springer, 2010, pp. 653-661.

[24] M. Gerritsma, R. Hiemstra, J. Kreeft, A. Palha, P.P. Rebelo, D. Toshniwal, The geometric basis of numerical methods, in: Spectral and High Order Methods for Partial Differential Equations, in: Lecture Notes in Computational Science and Engineering, vol. 95, Springer, 2013, pp. 17-35.

[25] J. Goedbloed, Some remarks on computing axisymmetric equilibria, Comput. Phys. Commun. 31 (1984) $123-135$.

[26] J.P. Goedbloed, R. Keppens, S. Poedts, Advanced Magnetohydrodynamics: With Applications to Laboratory and Astrophysical Plasmas, Cambridge University Press, 2010.

[27] W.J. Gordon, C.A. Hall, Transfinite element methods: blending-function interpolation over arbitrary curved element domains, Numer. Math. 21 (1973) 109-129.

[28] T. Görler, X. Lapillonne, S. Brunner, T. Dannert, F. Jenko, F. Merz, D. Told, The global version of the gyrokinetic turbulence code GENE, J. Comput. Phys. 230 (2011) 7053-7071.

[29] P.-A. Gourdain, J.-N. Leboeuf, R.Y. Neches, High-resolution magnetohydrodynamic equilibrium code for unity beta plasmas, J. Comput. Phys. 216 (2006) $275-299$.

[30] H. Grad, H. Rubin, Hydromagnetic equilibria and force-free fields, J. Nucl. Energy 7 (1958) 284-285.

[31] R. Gruber, F. Troyon, D. Berger, L. Bernard, S. Rousset, R. Schreiber, W. Kerner, W. Schneider, K. Roberts, ERATO stability code, Comput. Phys. Commun. 21 (1981) 323-371.

[32] H. Heumann, J. Blum, C. Boulbe, B. Faugeras, G. Selig, J.-M. Ané, S. Brémond, V. Grandgirard, P. Hertout, E. Nardon, Quasi-static free-boundary equilibrium of toroidal plasma with CEDRES++: computational methods and applications, J. Plasma Phys. (2015) 1-35.

[33] F.L. Hinton, R.D. Hazeltine, Theory of plasma transport in toroidal confinement systems, Rev. Mod. Phys. 48 (1976) 239-308.

[34] S.P. Hirshman, S.C. Jardin, Two-dimensional transport of tokamak plasmas, Phys. Fluids 22 (1979) 731.

[35] E. Howell, C. Sovinec, Solving the Grad-Shafranov equation with spectral elements, Comput. Phys. Commun. 185 (2014) $1415-1421$.

[36] D. Humphreys, G. Ambrosino, P. de Vries, F. Felici, S.H. Kim, G. Jackson, A. Kallenbach, E. Kolemen, J. Lister, D. Moreau, A. Pironti, G. Raupp, O. Sauter, E. Schuster, J. Snipes, W. Treutterer, M. Walker, A. Welander, A. Winter, L. Zabeo, Novel aspects of plasma control in ITER, Phys. Plasmas 22 (2015) 021806.

[37] G.T.A. Huysmans, J.P. Goedbloed, W. Kerner, Isoparametric bicubic Hermite elements for solution of the Grad-Shafranov equation, Int. J. Mod. Phys. C 2 (1991) 371-376.

[38] J.M. Hyman, M. Shashkov, S. Steinberg, The numerical solution of diffusion problems in strongly heterogeneous non-isotropic materials, J. Comput. Phys. 132 (1997) 130-148.

[39] R. Imazawa, Y. Kawano, K. Itami, Meshless method for solving fixed boundary problem of plasma equilibrium, J. Comput. Phys. 292 (2015) 208-214.

[40] A.A. Ivanov, R.R. Khayrutdinov, S.Y. Medvedev, Y.Y. Poshekhonov, New adaptive grid plasma evolution code SPIDER, in: 32nd EPS Conference on Plasma Physics, 2005, pp. 2146-2149.

[41] S. Jardin, A triangular finite element with first-derivative continuity applied to fusion MHD applications, J. Comput. Phys. 200 (2004) 133-152.

[42] S.C. Jardin, Computational Methods in Plasma Physics, Chapman \& Hall / CRC Computational Science, CRC Press, 2010.

[43] F. Kikuchi, K. Nakazato, T. Ushijima, Finite element approximation of a nonlinear eigenvalue problem related to MHD equilibria, Jpn. J. Appl. Math. 1 (1984) 369-403.

[44] J. Kreeft, A. Palha, M. Gerritsma, Mimetic framework on curvilinear quadrilaterals of arbitrary order, Arxiv preprint, arXiv:1111.4304 [math.NA], 2011, 69 pp.

[45] X. Lapillonne, S. Brunner, T. Dannert, S. Jolliet, A. Marinoni, L. Villard, T. Görler, F. Jenko, F. Merz, Clarifications to the limitations of the s- $\alpha$ equilibrium model for gyrokinetic computations of turbulence, Phys. Plasmas 16 (2009) 032308.

[46] J. Lee, A. Cerfon, ECOM: a fast and accurate solver for toroidal axisymmetric MHD equilibria, Comput. Phys. Commun. 190 (2015) 72-88.

[47] X. Li, L.E. Zakharov, V.V. Drozdov, Edge equilibrium code for tokamaks, Phys. Plasmas 21 (2014) 012505.

[48] L.L. LoDestro, L.D. Pearlstein, On the Grad-Shafranov equation as an eigenvalue problem, with implications for q solvers, Phys. Plasmas 1 (1994) $90-95$.

[49] G.O. Ludwig, Direct variational solutions of the tokamak equilibrium problem, Plasma Phys. Control. Fusion 39 (1997) $2021-2037$.

[50] H. Lütjens, A. Bondeson, A. Roy, Axisymmetric MHD equilibrium solver with bicubic Hermite elements, Comput. Phys. Commun. 69 (1992) 287-298.

[51] H. Lütjens, A. Bondeson, O. Sauter, The CHEASE code for toroidal MHD equilibria, Comput. Phys. Commun. 97 (1996) $219-260$.

[52] C. Mattiussi, An analysis of finite volume, finite element, and finite difference methods using some concepts from algebraic topology, J. Comput. Phys. 133 (1997) 289-309. 
[53] S.P. Neuman, Theoretical derivation of Darcy's law, Acta Mech. 25 (1977) 153-170.

[54] A. Palha, M. Gerritsma, Mimetic least-squares spectral/hp finite element method for the Poisson equation, in: Large-Scale Scientific Computing, in: Lecture Notes in Computer Science, vol. 5910, Springer, 2010, pp. 662-670.

[55] A. Palha, M. Gerritsma, Spectral element approximation of the Hodge-^ operator in curved elements, in: Spectral and High Order Methods for Partial Differential Equations, in: Lecture Notes in Computational Science and Engineering, vol. 76, Springer, 2010, pp. $283-291$.

[56] A. Palha, P.P. Rebelo, R. Hiemstra, J. Kreeft, M. Gerritsma, Physics-compatible discretization techniques on single and dual grids, with application to the Poisson equation of volume forms, J. Comput. Phys. 257 (2014) 1394-1422.

[57] V. Parail, R. Albanese, R. Ambrosino, J.-F. Artaud, K. Besseghir, M. Cavinato, G. Corrigan, J. Garcia, L. Garzotti, Y. Gribov, F. Imbeaux, F. Koechl, C. Labate, J. Lister, X. Litaudon, A. Loarte, P. Maget, M. Mattei, D. McDonald, E. Nardon, G. Saibene, R. Sartori, J. Urban, Self-consistent simulation of plasma scenarios for ITER using a combination of 1.5D transport codes and free-boundary equilibrium codes, Nucl. Fusion 53 (2013) 113002.

[58] P.B. Parks, M.J. Schaffer, Analytical equilibrium and interchange stability of single- and double-axis field-reversed configurations inside a cylindrical cavity, Phys. Plasmas 10 (2003) 1411-1423.

[59] A. Pataki, A.J. Cerfon, J.P. Freidberg, L. Greengard, M. O’Neil, A fast, high-order solver for the Grad-Shafranov equation, J. Comput. Phys. 243 (2013) $28-45$.

[60] G.V. Pereverzev, P.N. Yushmanov, ASTRA Automated System for TRansport Analysis in a Tokamak, Tech. rep., IPP, 2002.

[61] P.P. Rebelo, A. Palha, M. Gerritsma, Mixed mimetic spectral element method applied to Darcy's problem, in: Spectral and High Order Methods for Partial Differential Equations - ICOSAHOM 2012, in: Lecture Notes in Computational Science and Engineering, vol. 95, Springer, 2014, pp. 373-382.

[62] N. Robidoux, Polynomial histopolation, superconvergent degrees of freedom, and pseudospectral discrete Hodge operators, Unpublished: http://people. math.sfu.ca/ nrobidou/public_html/prints/histogram/histogram.pdf.

[63] N. Robidoux, S. Steinberg, A discrete vector calculus in tensor grids, Comput. Methods Appl. Math. 11 (2011) 23-66.

[64] A. Saitoh, T. Itoh, N. Matsui, A. Kamitani, H. Nakamura, Application of collocation meshless method to eigenvalue problem, Plasma Fusion Res. 7 (2012) 2406096.

[65] V.D. Shafranov, Magnetohydrodynamical equilibrium configurations, Sov. Phys. JETP 6 (1958) 545-554.

[66] C. Sovinec, A. Glasser, T. Gianakon, D. Barnes, R. Nebel, S. Kruger, D. Schnack, S. Plimpton, A. Tarditi, M. Chu, N. Team, Nonlinear magnetohydrodynamics simulation using high-order finite elements, J. Comput. Phys. 195 (2004) 355-386.

[67] T. Takeda, S. Tokuda, Computation of MHD equilibrium of tokamak plasma, J. Comput. Phys. 93 (1991) 1-107.

[68] E. Tonti, On the formal structure of physical theories, Tech. rep., Italian National Research Council, 1975.

[69] E. Tonti, The Mathematical Structure of Classical and Relativistic Physics, Birkhäuser, 2013.

[70] E. Tonti, Why starting from differential equations for computational physics?, J. Comput. Phys. 257 (2014) $1260-1290$.

[71] L.E. Zakharov, A. Pletzer, Theory of perturbed equilibria for solving the Grad-Shafranov equation, Phys. Plasmas 6 (1999) 4693. 